\title{
Non-uniqueness of the Leray-Hopf solutions in the hyperbolic setting
}

\author{
Chi Hin Chan and Magdalena Czubak \\ Communicated by Boris Khesin, received January 28, 2013.
}

\begin{abstract}
The Leray-Hopf solutions to the Navier-Stokes equation are known to be unique on $\mathbb{R}^{2}$. We show the uniqueness of the Leray-Hopf solutions breaks down on $\mathbb{H}^{2}\left(-a^{2}\right)$, the two dimensional hyperbolic space with constant sectional curvature $-a^{2}$. We also obtain a corresponding result on a more general negatively curved manifold for a modified geometric version of the Navier-Stokes equation. Finally, as a corollary we also show a lack of the Liouville theorem in the hyperbolic setting both in two and three dimensions.
\end{abstract}

\section{Contents}

\begin{tabular}{|c|c|}
\hline \multicolumn{2}{|l|}{ 1. Introduction } \\
\hline Acknowledgements & 54 \\
\hline 2. Preliminaries & 54 \\
\hline 3. Exponential decav of the gradient of bounded harmonic functions & 62 \\
\hline 4. The proof that $\left\|\nabla|\nabla F|^{2}\right\|_{I 1\left(\mathbb{1} 2\left(-\alpha^{2}\right)\right)}$ is finite & 64 \\
\hline 5. Finite Dissipation & 69 \\
\hline 6. Proofs of the main results & 71 \\
\hline References & 75 \\
\hline
\end{tabular}

\section{Introduction}

We investigate the impact of geometry on the Leray-Hopf solutions to the Navier-Stokes equation. More precisely, we consider the Navier-Stokes equation on negatively curved manifolds and present how the negative scalar curvature causes the break down of the uniqueness of the Leray-Hopf solutions.

Before we state the main results, we survey some historical background from

2000 Mathematics Subject Classification. 76D05;

Key words and phrases. Navier-Stokes, Leray-Hopf, non-uniqueness, hyperbolic space, Liouville theorem. 
both geometric analysis, and the regularity theory for the Navier-Stokes equation in the usual Euclidean setting.

1.1. Regularity theory for the Navier-Stokes equation on $\mathbb{R}^{n}$. The Navier-Stokes equation on the Euclidean space $\mathbb{R}^{n}$ is given by

$$
\begin{aligned}
\partial_{t} u-\Delta u+u \cdot \nabla u+\nabla P & =0, \\
\operatorname{div} u & =0 .
\end{aligned}
$$

Long time ago, for the dimensions $n=2$ and $n=3$, Leray [20] and Hopf 8 established the existence of global weak solutions

$$
u \in L^{\infty}\left(0, \infty ; L^{2}\left(\mathbb{R}^{n}\right)\right) \cap L^{2}\left(0, \infty ; \dot{H}^{1}\left(\mathbb{R}^{n}\right)\right)
$$

. Due to their work, we now have the following general existence result,

Theorem 1.1 (Leray-Hopf weak solutions [20, 8]). Let $n=2$ or 3 . Given any initial datum $u_{0} \in L^{2}\left(\mathbb{R}^{n}\right)$, there exists at least one $\mathbb{R}^{n}$-valued function $u \in$ $L^{\infty}\left(0, \infty ; L^{2}\left(\mathbb{R}^{n}\right)\right) \cap L^{2}\left(0, \infty ; H^{1}\left(\mathbb{R}^{n}\right)\right)$ which satisfies the following properties

- For any $\phi=\left(\phi_{1}, \ldots, \phi_{n}\right) \in C_{c}^{\infty}\left((0, \infty) \times \mathbb{R}^{n}\right)$ with $\operatorname{div} \phi=0$, we have

$$
\int_{0}^{\infty} \int_{\mathbb{R}^{n}}-u \cdot \partial_{t} \phi+\sum_{i, j}\left(\partial_{j} \phi_{i}\right)\left(\partial_{j} u_{i}\right)-\sum_{i, j}\left(\partial_{j} \phi_{i}\right)\left(u_{i} u_{j}\right) d x d t=0 .
$$

- For every $t \geq 0, u$ satisfies the following global energy inequality

$$
\int_{\mathbb{R}^{n}}|u(t, x)|^{2} d x+2 \int_{0}^{t} \int_{\mathbb{R}^{n}}|\nabla u|^{2} d x d s \leq \int_{\mathbb{R}^{n}}\left|u_{0}\right|^{2} d x .
$$

- $u(0, \cdot)$ coincides with the initial datum $u_{0}$ in the sense that

$$
\lim _{t \rightarrow 0^{+}}\left\|u(t, \cdot)-u_{0}\right\|_{L^{2}\left(\mathbb{R}^{n}\right)}=0 .
$$

Solutions $u \in L^{\infty}\left(0, \infty ; L^{2}\left(\mathbb{R}^{n}\right)\right) \cap L^{2}\left(0, \infty ; \dot{H}^{1}\left(\mathbb{R}^{n}\right)\right)$ satisfying the global energy inequality are hence referred to as the Leray-Hopf solutions, and have historically served as a foundation for further work in the regularity theory for $\mathrm{N}-\mathrm{S}_{\mathbb{R}^{n}}$.

The regularity of the Leray-Hopf solutions on $\mathbb{R}^{2}$ greatly differs from the corresponding regularity problem on $\mathbb{R}^{3}$. Indeed, the smoothness and uniqueness of the Leray-Hopf solutions for $\mathrm{N}-\mathrm{S}_{\mathbb{R}^{2}}$ is known and can be found in the work of Ladyzhenskaya 18. As a sharp contrast, the regularity and uniqueness of solutions to the $\mathrm{N}-\mathrm{S}_{\mathbb{R}^{3}}$ equation is a long standing open problem although due to the concentrated efforts by generations of PDE specialists, there has been a significant progress in this area.

Because of the limitation of space, we only mention some typical regularity criteria for the Leray-Hopf solutions to $\mathrm{N}-\mathrm{S}_{\mathbb{R}^{3}}$. We also note that one of the goals of this discussion is to illustrate why there is more focus on the question of regularity than that of the uniqueness.

Now, the first significant effort to break the silence since the fundamental work of Leray and Hopf, was made in 1960's through the efforts of Prodi [25, Serrin [28, and Ladyzhenskaya [1] leading to the following regularity and uniqueness result (for more historical remarks see for instance 12]).

Theorem 1.2. [Prodi, Serrin, Ladyzhenskaya] Let $u \in L^{\infty}\left(0, T ; L^{2}\left(\mathbb{R}^{3}\right)\right) \cap$ $L^{2}\left(0, T ; \dot{H}^{1}\left(\mathbb{R}^{3}\right)\right)$ be a Leray-Hopf weak solution to $\mathrm{N}-\mathrm{S}_{\mathbb{R}^{3}}$, which satisfies the additional condition that $u \in L^{p}\left(0, T ; L^{q}\left(\mathbb{R}^{3}\right)\right)$, for some $p, q$ satisfying $\frac{2}{p}+\frac{3}{q}=1$, 
with $q>3$. Then, $u$ is smooth on $(0, T] \times \mathbb{R}^{3}$, and $u$ is uniquely determined in the following sense

- suppose $v \in L^{\infty}\left(0, T ; L^{2}\left(\mathbb{R}^{3}\right)\right) \cap L^{2}\left(0, T ; H^{1}\left(\mathbb{R}^{3}\right)\right)$ is another Leray-Hopf weak solution such that $u(0, \cdot)=v(0, \cdot)$. Then, it follows that $u=v$ on $(0, T] \times \mathbb{R}^{3}$.

Here, let us briefly mention why the case of $q=3$ was not included in Theorem 1.2 First, it is well known that a solution $\theta:[0, T) \times \mathbb{R}^{3} \rightarrow \mathbb{R}$ to the heat equation arising from any initial datum $\theta_{0} \in L^{3}\left(\mathbb{R}^{3}\right)$ satisfies the following estimate for any pair of indices $p, q$ with $\frac{2}{p}+\frac{3}{q}=1$ and $q>3$ (see [12 Appendix])

$$
\|\theta\|_{L^{p}\left(0, T ; L^{q}\left(\mathbb{R}^{3}\right)\right)} \leq C(q)\left\|u_{0}\right\|_{L^{3}\left(\mathbb{R}^{3}\right)},
$$

where $C_{q}$ depends only on $q$. So, in some sense, the extra condition as imposed on the Leray-Hopf solution $u$ in Theorem 1.2 ensures that the qualitative behavior of the Leray-Hopf solution $u$ would be a slight perturbation of solutions of the heat equation. Second reason is that the $L_{t}^{p} L_{x}^{q}$ norm of the solution $u$ under the integral condition as promised in Theorem 1.2 shrinks to zero under the natural scaling $u_{\epsilon}(t, x)=\epsilon u\left(\epsilon^{2} t, \epsilon x\right)$ as $\epsilon \rightarrow 0$. However, this is no longer valid in the borderline case of $L^{\infty}\left(0, T ; L^{3}\left(\mathbb{R}^{3}\right)\right)$. This partially explains the long delay in the settlement of this exceptional case of $u \in L^{\infty}\left(0, T ; L^{3}\left(\mathbb{R}^{3}\right)\right)$, which was finally established in the recent work of Escauriaza, Seregin, and Šverák [12.

Before we close our discussion let us mention that one of the working principles in the regularity theory of Navier-Stokes equation on $\mathbb{R}^{3}$ is (more or less) to reduce the situation under consideration (say $u \in L^{\infty}\left(L^{3}\right)$ in the case of [12) to the regime which is covered by Theorem 1.2 Once this can be achieved, then the uniqueness of the solution would come for free, due to the uniqueness claim in Theorem 1.2 This explains to some extent the fact that regularity issue is more of a focus than the uniqueness issue in the regularity theory for Navier-Stokes equation in the $\mathbb{R}^{3}$ setting. However, as is well-known, the weak formulation for Leray-Hopf weak solutions to the Navier-Stokes equation on $\mathbb{R}^{3}$ only gives the natural bound $u \in L^{p}\left(0, \infty ; L^{q}\left(\mathbb{R}^{3}\right)\right)$, with indices $p, q$ satisfying $\frac{2}{p}+\frac{3}{q}=\frac{3}{2}$. One readily sees that there is a significant gap between the natural bound offered by the weak formulation and the condition required by Theorem 1.2 and it is unclear how to make a link between them. See again the introduction of $\mathbf{1 2}$ for a discussion about this point, and for further developments, we refer our readers to a piece of recent work by Vasseur 33.

1.2. Navier Stokes equation on a Riemannian manifold. Historically speaking, the correct form of the Navier-Stokes equation in the Riemannian manifold setting was first obtained by Ebin and Marsden [5. They consider compact Riemannian, oriented, $n$-dimensional manifolds both with and without boundary. Moreover, they remark that the derivation of the correct equations assumes that the manifold in question is Einstein: Ric $=\lambda g$, for some constant $\lambda$ where Ric is the Ricci tensor, and $g$ is the Riemannian metric. We note, this is in particular true of space forms, where Ric $=(n-1) K_{M} g$ (see Section 2.1 below).

According to [5] the ordinary Laplacian should be replaced by the following 
operator in the formulation of the Navier-Stokes equation on a Riemannian manifold

$$
L=2 \operatorname{Def}^{*} \text { Def }=\bar{\nabla}^{*} \bar{\nabla}+d d^{*}-\mathrm{Ric}=\left(d d^{*}+d^{*} d\right)+d d^{*}-2 \mathrm{Ric},
$$

where Def and Def* are the deformation tensor and its adjoint respectively, $\bar{\nabla}$ stands for the induced Levi-Civita connection on the cotangent bundle $T^{*} M, \bar{\nabla}^{*} \bar{\nabla}$ is the Bochner Laplacian, with $\bar{\nabla}^{*}$ the adjoint operator associated to $\bar{\nabla},\left(d d^{*}+d^{*} d\right)=$ $-\Delta$ stands for the Hodge Laplacian with $d^{*}$ the formal adjoint of the exterior differential operator $d$, and Ric is the Ricci operator (see Sections 2.1] and 2.6] for definitions and 4, 30 for a further discussion of the deformation tensor).

We first remark that the operator $L$, as given in expression (1.2), sends sections of $T^{*} M$ into sections of $T^{*} M$. Hence, it is more convenient to formulate the NavierStokes equation on a Riemannian manifold $M$ in terms of sections of $T^{*} M$ instead of vector fields on $M$. As a result, the usual convection term $\nabla_{u} u$ in terms of vector fields also has to be rewritten. There is a natural correspondence between vector fields and 1-forms (see Section 2.1), which produces the term $\bar{\nabla}_{U} U^{*}$, where $U$ is the unique vector field corresponding to a 1 -form $U^{*}$.

In summary, the solutions that we consider in this article will be differential 1-forms $U^{*} \in C^{\infty}\left(M ; T^{*} M\right)$ satisfying the following differential equation

$$
\begin{aligned}
& \partial_{t} U^{*}+L\left(U^{*}\right)+\bar{\nabla}_{U} U^{*}+d P=0, \\
& d^{*} U^{*}=0 .
\end{aligned}
$$

where $P$ is a scalar function on $M$. Using the fact that $U^{*}$ is divergence free we can further rewrite the equations as follows

$$
\begin{aligned}
\partial_{t} U^{*}-\Delta U^{*}+\bar{\nabla}_{U} U^{*}-2 \operatorname{Ric}\left(U^{*}\right)+d P & =0, \\
d^{*} U^{*} & =0 .
\end{aligned}
$$

which is the main equation that we study.

Arguably a less natural equation to consider is the one without the Ricci operator. We refer to it as the modified Navier-Stokes equation on $M$ and record it here

$$
\begin{aligned}
\partial_{t} U^{*}-\Delta U^{*}+\bar{\nabla}_{U} U^{*}+d P & =0 . \\
d^{*} U^{*} & =0
\end{aligned}
$$

It might be considered less natural from the point of view of the derivation of the Navier-Stokes equation performed in $[\mathbf{5}$. Nevertheless, it has been studied for example in [3, 11, 10, 31. Moreover, we consider it in this paper as well, because we would like to present how a more general manifold than just a space form can influence the behavior of solutions (we explain this more below). At the same time, in the context of this paper, the definition of the dissipation appears to be more natural using the deformation tensor than without it, and hence this seems to confirm that the equation $\mathrm{N}_{-} \mathrm{S}_{M}$ is indeed more natural than (1.3) (also see remark 1.8).

Since 2 Def* $^{*}$ Def $U^{*}$ plays now the role of the viscosity, the global energy inequality becomes

$$
\int_{M}\left|U^{*}\right|^{2}(t, x)+4 \int_{0}^{t} \int_{M} \bar{g}\left(\operatorname{Def} U^{*}, \operatorname{Def} U^{*}\right) d s \leq \int_{M}\left|U^{*}(0)\right|^{2},
$$


where $\bar{g}(\cdot, \cdot)$ stands for the inner product structure on the bundle $T^{*} M \otimes T^{*} M$ induced by the Riemannian metric $g(\cdot, \cdot)$ on $M$ (see Section 2.11). From now on, when we refer to energy we mean the first term in (1.4)

$$
\int_{M}\left|U^{*}\right|^{2}(t, x)
$$

and by dissipation we mean

$$
4 \int_{0}^{t} \int_{M} \bar{g}\left(\operatorname{Def} U^{*}, \operatorname{Def} U^{*}\right)
$$

We now mention some of the previous results on a Riemannian manifold (for more see 4 and references therein). Priebe [24] appears to be the first one to follow [5] and use the correct version of the equations $\mathrm{N}^{-\mathrm{S}_{M}}$ instead of (1.3). 24]. also assumes compactness of $M$ and works on manifolds with boundary. Mazzucato considers Besov-Morrey spaces on compact, smooth, Riemannian manifolds without boundary 21. Mitrea and Taylor study the Stokes and Navier-Stokes equations on bounded Lipschitz domains in Riemannian manifolds [22. Dindoš and Mitrea 4] address the linearized version of the stationary Navier-Stokes equation on a subdomain of a compact Riemannian manifold. In fact, we have not been able to find any results for non-compact manifolds except for the result of Q.S. Zhang [35. In [35] the author shows the ill-posedness of the weak solution with finite $L^{2}$ norm on a connected sum of two copies of $\mathbb{R}^{3}$. Hence the topology of the underlying manifold seems to play a role. In this paper, we take a geometric point of view, and also consider the dissipation term.

We are now ready to state our main results.

\subsection{Statements of the results and discussion of the proofs.}

Theorem 1.3 (Non-uniqueness of $\mathrm{N}-\mathrm{S}_{\mathbb{H}^{2}\left(-a^{2}\right)}$ ). Let $a>0$. Then, $\mathrm{N}-\mathrm{S}_{\mathbb{H}^{2}\left(-a^{2}\right)}$ is ill-posed in the following sense: we can find smooth divergence free initial data $u_{0} \in L^{2}\left(\mathbb{H}^{2}\left(-a^{2}\right)\right)$ such that there exist infinitely many smooth time dependent solutions, which have finite energy (1.5), finite dissipation (1.6), and satisfy the global energy inequality (1.4).

REMARK 1.4. We emphasize this is contrary to what is known about the solutions on $\mathbb{R}^{2}$. In addition, one consequence of Theorem 1.3 is that the conventional notion of the Leray-Hopf solutions might not be the proper foundation for the study of solutions on $\mathbb{H}^{2}\left(-a^{2}\right)$. The question of what happens on $\mathbb{H}^{3}\left(-a^{2}\right)$ is open. The lack of a definite answer at this point is mainly due to the specific form of the techniques we use in this paper (see remarks below). It is an interesting question to see if perhaps the techniques could eventually be extended/modified to give some insight into the corresponding question in 3 dimensions 1

CoROLlary 1.5 (Lack of the Liouville theorem for space forms). Let $n \geq 2$, and $a>0$, then there exist global in time, nontrivial bounded solutions of $\mathrm{N}-\mathrm{S}_{H^{n}\left(-a^{2}\right)}$.

\footnotetext{
${ }^{1}$ Since the completion of this work in 2010, Khesin and Misiołek have shown in their recent and elegant paper $\mathbf{1 5}$ that the form of the solutions we choose could not lead to non-uniqueness in $3 \mathrm{D}$.
} 
REMARK 1.6. The proof of Corollary [1.5] and Corollary 1.9 below follows trivially from the proofs of their theorems. Moreover, it does not require any of the delicate estimates developed in this paper. As such it is just a by-product of the main results, and we only include it here for completeness, and because of the general importance the Liouville theorems play in the subject of the Navier-Stokes equation. See Section 6.3 for motivation and some background.

If one decides to omit the Ricci term from $\mathrm{N}_{-} \mathrm{S}_{M}$, and consider (1.3) instead, we can also have a non-uniqueness result on a more general negatively curved Riemannian manifold than just $\mathbb{H}^{2}\left(-a^{2}\right)$.

Theorem 1.7. Let $a, b>0$ be such that $\frac{1}{2} b<a \leq b$, and let $M$ be a simply connected, complete 2-dimensional Riemannian manifold with sectional curvature satisfying $-b^{2} \leq K_{M} \leq-a^{2}$. Then, (1.3) is ill-posed in the following sense: we can find smooth divergence free initial data $u_{0} \in L^{2}(M)$ such that there exist infinitely many smooth time dependent solutions, which have finite energy (1.5), finite dissipation (1.6), and satisfy the global energy inequality (1.4).

REMARK 1.8. Note that using (1.6) for dissipation and (1.4) for the global energy inequality may not be the natural choice since the deformation tensor does not make an appearance in equation (1.3). On the other hand it might be even less natural to redefine these concepts and the global energy inequality. However, if one insists on redefining, then the only natural candidate to replace the dissipation would be:

$$
\int_{0}^{t} \int_{M} g\left(d U^{*}, d U^{*}\right)
$$

which in our context would be trivial (see below). After that, the possible candidate would be

$$
\int_{0}^{t} \int_{M} g\left(\bar{\nabla} U^{*}, \bar{\nabla} U^{*}\right)
$$

which is not natural since it leads to the Bochner Laplacian and not the Hodge Laplacian. Hence we believe it is best to still use (1.6) for dissipation even in this context.

COROLlary 1.9 (Lack of the Liouville theorem in the hyperbolic setting). Let $n \geq 2$, and $b \geq a>0$, and let $M$ be a simply connected, complete $n$-dimensional Riemannian manifold with sectional curvature satisfying $-b^{2} \leq K_{M} \leq-a^{2}$. Then there exist global in time, nontrivial bounded solutions of (1.3).

REMARK 1.10. Note, the lower bound $\frac{1}{2} b<a$ is no longer required in the corollary. See the discussion below for why the lower bound is present in Theorem 1.7 which also explains why we do not need it in Corollary 1.9

REMARK 1.11. [Non-uniqueness of the Euler equation on $M$ ]. We end the statements of the results by including the following remark about the Euler equation on a Riemannian manifold $M$. Let $b \geq a>0$. Let $M$ be a simply connected, complete 2-dimensional Riemannian manifold with sectional curvature satisfying $-b^{2} \leq K_{M} \leq-a^{2}$. Then the non-unique solutions we construct for the N-S could also provide non-unique finite energy solutions to the Euler equation

$$
\begin{aligned}
\partial_{t} U^{*}+\bar{\nabla}_{U} U^{*}+d P & =0, \\
d^{*} U^{*} & =0 .
\end{aligned}
$$


However due to the construction, as one can check, the solutions would not be conserving the energy.

The above results are based on the abundance of the nontrival bounded harmonic functions in the hyperbolic setting. Such abundance is ensured by the works of Anderson 1 and Sullivan [29. Our idea of trying to benefit from them was inspired by a remark of Tsai [32, Remark 5.4]. 32 eliminates a possibility of self-similar solutions to $\mathrm{N}-\mathrm{S}_{\mathbb{R}^{3}}$ (which merely would satisfy the local energy inequality) by showing that existence of the self-similar solutions is equivalent to solving a certain stationary system. Without assuming enough decay, one could construct nontrival solutions of the system in question in the form of $U=\nabla F$, and $P=-\frac{1}{2}|U|^{2}-c y \cdot U$, where $F$ is a harmonic function on $\mathbb{R}^{3}$ and $c>0$. In our case, due to [1] and 29] we have a plethora of nontrival bounded harmonic functions, which gives us a basis for this article.

The solution pairs $\left(U^{*}, P\right)$ we consider have the following form

$$
\begin{aligned}
U^{*} & =\psi(t) d F, \\
P & =-\partial_{t} \psi(t) F-\frac{1}{2} \psi^{2}(t)|d F|^{2}-2 a^{2} \psi(t) F,
\end{aligned}
$$

where $\psi(t)=\exp \left(-\frac{A t}{2}\right)$ for any $A \geq 4 a^{2}$, and $F$ is a nontrival bounded harmonic function on $\mathbb{H}^{2}\left(-a^{2}\right)$. Verifying that $\left(U^{*}, P\right)$ solves $\mathrm{N}_{-} \mathrm{S}_{\mathbb{H}^{2}\left(-a^{2}\right)}$ is simple when we use Hodge theory (see Sections [2.6 and [6). In fact, taking solutions of the form $\psi(t) \nabla F$ seems to be a well known convention, and we just happened to learn about it from [32 (but also see [35). Hence the heart of the matter and the main contribution is in proving that the solutions in that form have a finite energy and a finite dissipation.

Before we proceed any further, we remark here that the differential geometric work in [1, 29, 2 ensures the existence of nontrival bounded harmonic functions on a more general negatively curved Riemannian manifold with suitable lower and upper bounds imposed on the sectional curvature. On the other hand, the existence of nontrival bounded harmonic function on $\mathbb{H}^{n}(-1)$ is an old classical result obtained through an integral representation formula with an explicit Poisson kernel on the Poincare ball model for the space form $\mathbb{H}^{n}(-1)$ (for more details see the work of Hua 9]). However, such classical approach relies heavily on the explicit formula of the Poisson kernel derived from the group of isometries of the space form $\mathbb{H}^{n}(-1)$. It seems that, as compared with the differential geometric approach of 1, 29, 2, such classical approach does not reveal the role played by the negative sectional curvature of the hyperbolic manifold in the existence of nontrival bounded harmonic functions on $\mathbb{H}^{n}(-1)$.

The last remark may explain why the proper generalization of the above mentioned classical result to the more general setting of the negatively curved Riemannian manifolds was only established in the more recent works 1, 29, 2]. Since we intend to show not only the break down of the uniqueness of Leray-Hopf solutions in the hyperbolic space setting, but more importantly the decisive role played by the negative sectional curvature of a hyperbolic manifold in causing such a breakdown, we will unconditionally choose the differential geometric framework as established in 1, 29, 2] as the basic ground in this paper.

Moreover, since the differential geometric machinery as demonstrated in 10, 29, 2 is designed to establish the existence of bounded nonconstant harmonic function 
on a general negatively curved Riemannian manifold which lacks the homogeneity property enjoyed by the space form $\mathbb{H}^{2}\left(-a^{2}\right)$, the only best way to justify the use of such differential geometric machinery is to cast our theorems, lemmas, propositions in the most general setting of a negatively curved Riemannian manifold at the starting point of the paper. However, we slowly narrow down our setting by imposing further restrictions on our results whenever such restrictions are needed in proving the finite integral of a certain function or in handling the extra Ric term in the formulation of the Navier-Stokes equation. The reason is that in the case of the space form Ric is just an idenity times a constant (see 2.12) as opposed to the general expression in (2.10). Thus when we define the pressure $P$ in (1.7), we can introduce $-2 a^{2} \psi(t) F$, so when we take the exterior derivative $d$ in the equation, it will cancel exactly with the 2 Ric term. It is not obvious how to do this for a more general manifold.

As stated in Theorem 1.7 our non-uniqueness result also holds for a more general negatively curved Riemannian manifold with the lower bound $-b^{2}$ and the upper bound $-a^{2}$ of the sectional curvature satisfying $0<\frac{b}{2}<a \leq b$, provided if the extra Ric term in the Navier-Stokes equation is dropped. Indeed, the final restriction to the space form $\mathbb{H}^{2}\left(-a^{2}\right)$ is required only because of the presence of -2 Ric in the formulation of the Navier-Stokes equation $\mathrm{N}_{-} \mathrm{S}_{M}$.

Now, we explain our strategy in establishing the finite energy and the finite dissipation of the time dependent velocity field $U^{*}=\psi(t) d F$ as given in equation (1.7). We start our discussion by saying that our exposition is based on the material in the second chapter of the book 27 by R. Schoen and S.-T. Yau. In the first section of the second chapter of [27, one sees that, with the prescribed function $\phi \in C^{1}(S(\infty)$ ) given on the geometric boundary $S(\infty)$ (see Section 2.4) attached to the $n$-dimensional complete, simply connected Riemannian manifold $M$ with sectional curvature satisfying $-b^{2} \leq K_{M} \leq-a^{2}<0$, the bounded harmonic function $F$ on $M$ which satisfies the Dirichlet boundary condition $\left.F\right|_{S(\infty)}=\phi$ is sought after by means of creating two barrier functions $\bar{\phi}-\alpha e^{-\delta \rho}$ and $\bar{\phi}+\alpha e^{-\delta \rho}$, where $\rho$ stands for the distance function on $M$ from a selected base point $O$ in $M$, and $\bar{\phi}$ is given by (2.31). These two functions serve as the lower bound and upper bound for $F$ (also see Section 2.5 and 2.2). This is done in the spirit of the classical Perron's method. However, such an application requires the subharmonicity of $\bar{\phi}-\alpha e^{-\delta \rho}$ and the superharmonicity of $\bar{\phi}+\alpha e^{-\delta \rho}$, whose validity critically depends on the following two facts (for details, see [27])

- Laplace comparison theorem: If $K_{M} \leq-a^{2}$, then, the Laplacian of the distance function $\rho$ (from a selected base point $O$ in $M$ ) satisfies $\Delta \rho \geq$ $(n-1) a \operatorname{coth}(a \rho) \geq(n-1) a$.

- the smooth function $\bar{\phi}$ is constructed in a specific way so that we have $\left.\bar{\phi}\right|_{S(\infty)}=\phi$ and that the oscillation of $\bar{\phi}$ over any geodesic ball $B_{x}(1)$ in $M$ has exponential decay of order $e^{-a \rho(x)}$, for any $x \in M$.

Due to the above two facts, it can be deduced that the choice of the $\delta>0$, which ensures $\Delta\left[\bar{\phi}-\alpha e^{-\delta \rho}\right] \geq 0$ and $\Delta\left[\bar{\phi}+\alpha e^{-\delta \rho}\right] \leq 0$ (and hence the success of the Perron's method), has to satisfy the constraint $\delta<a$ (see Section 2.5.1).

Based on what we learn from the above construction of the bounded nontrival harmonic function $F$ on $M$ we employ, in Section 3, the gradient estimate for harmonic functions due to S.-T. Yau 34 to show that the decay rate for $|\nabla F|(x)$ as $\rho(x)$ approaches infinity is at least of the order $e^{-\delta \rho(x)}$, for any $\delta<a$. That is 
we have

$$
|\nabla F| \leq C e^{-\delta \rho} .
$$

This result has nothing to do with the Navier-Stokes equation, and it might be of independent interest. Nevertheless, we want to mention that with the hindsight from one of the two Harnack's inequalities as established in the second chapter of 27. one can argue that the exponential decay is more or less expected and may not be surprising. We believe that it is possible that such an exponential decay could be known to the researchers working in geometric analysis. But in any case, we give a simple proof of it in Section 3 .

Next we note that the exponential decay (1.8) not only gives, in the special case of the two dimensional space form $\mathbb{H}^{2}\left(-a^{2}\right)$, the $L^{2}$-finite property of $|\nabla F|$ on $\mathbb{H}^{2}\left(-a^{2}\right.$ ) (and hence the finite energy property of the velocity field $U^{*}=\psi(t) d F$ as given in (1.7)), but also demonstrates the limitation which prevents us to draw the same $L^{2}$-finite property of $|\nabla F|$ in the setting of the 3-dimensional space form $\mathbb{H}^{3}\left(-a^{2}\right)$. This limitation mainly comes from the fact that $\operatorname{osc}_{B_{x}(1)} \bar{\phi}$ only has exponential decay of order $e^{-a \rho(x)}$, which prevents us from choosing $\delta>0$ larger than $a$ in the Perron's method; yet the growth rate of the volume form on $\mathbb{H}^{3}\left(-a^{2}\right)$ is exactly of the order $\frac{1}{a^{2}} \sinh ^{2}(a \rho)$. For the first time, we encounter an obstacle which forces us to restrict our theory only to the case of the 2-dimensional negatively curved Riemannian manifold $M$ with $-b^{2} \leq K_{M} \leq-a^{2}<0$.

We observe that, up to this point, the lower bound condition $K_{M} \geq-b^{2}$ has not been involved in the big picture yet. However one does eventually have to pay a special attention to the relative largeness of $b$ when compared with $a$ since the lower bound $K_{M} \geq-b^{2}$ of the sectional curvature determines the growth rate of the volume form of $M$ via the comparison theorem for Jacobi fields in differential geometry. More precisely, with the condition $K_{M} \geq-b^{2}$ imposed, the growth rate of the volume form of the 2-dimensional negatively curved manifold $M$ is at most $\frac{1}{b} \sinh (b \rho)$. Yet, again, the decay rate of $|\nabla F|$ is of the order $e^{-\delta \rho}$, with any $\delta<a$. As a result, the survival of the property $\|\nabla F\|_{L^{2}(M)}<\infty$ critically depends on the competition between the decay rate $e^{-\delta \rho}$ of $|\nabla F|$ and the (possible) worst growth rate $\frac{1}{b} \sinh (b \rho)$ of the volume form of $M$. This fully explains the need to further restrict our setting by imposing the condition $0<\frac{b}{2}<a \leq b$, so that the parameter $\delta$ can fit within the range of $\frac{b}{2}<\delta<a$, which is enough to ensure the survival of the $L^{2}$-finite property of $|\nabla F|$ on the 2-dimensional negatively curved manifold $M$.

Once the $L^{2}$-finite property of $|\nabla F|$ is established for the 2-dimensional Riemannian manifold satisfying $-b^{2} \leq K_{M} \leq-a^{2}$ and $0<\frac{b}{2}<a \leq b$, we proceed to show the finite dissipation of $U^{*}=\psi(t) d F$ under the same setting in Sections 4 and 5. In regard to this, our basic idea lies in the structure of the following important formula in differential geometry 27

$\Delta\left(|\nabla F|^{2}\right)(x)=2 \bar{g}(\bar{\nabla}(\nabla F), \bar{\nabla}(\nabla F))(x)+2 \sum \partial_{i} F(x) \partial_{i}(\Delta F)(x)+2 R i c(\nabla F, \nabla F)(x)$.

The formula is obtained by performing a calculation with respect to the normal geodesic coordinates about the selected point $x$, and it holds for a general Riemannian manifold $M$. Since $\Delta\left(|\nabla F|^{2}\right)=\operatorname{div}\left\{\nabla\left(|\nabla F|^{2}\right)\right\}$, if we can show that the $L^{1}$-norm of $\left.|\nabla| \nabla F\right|^{2} \mid$ is finite, then we immediately have $\int_{M} \Delta\left(|\nabla F|^{2}\right)=0$. Hence, 
it follows from (1.9) that (see Proposition 5.3 for the technical details)

$$
\int_{M} \bar{g}(\bar{\nabla}(\nabla F), \bar{\nabla}(\nabla F))=-\int_{M} \operatorname{Ric}(\nabla F, \nabla F) \leq b^{2} \int_{M}|\nabla F|^{2},
$$

which with Corollary 5.2 gives the finiteness of the dissipation

$$
\int_{M} \bar{g}(\operatorname{Def} U, \operatorname{Def} U)=\int_{M} \bar{g}(\bar{\nabla} U, \bar{\nabla} U)=[\psi(t)]^{2} \int_{M} \bar{g}(\bar{\nabla}(\nabla F), \bar{\nabla}(\nabla F)),
$$

as required in the Leray-Hopf formulation.

Next, the $L^{1}$-finite property of $\left.|\nabla| \nabla F\right|^{2} \mid$ is established with the assistance of a covering Lemma 4.1] Due to the fact that $\operatorname{Ric}(\nabla F, \nabla F) \geq-b^{2}|\nabla F|^{2}$ and that $\Delta F=0$, formula (1.9) ensures the subharmonicity of $|\nabla F|^{2}+A e^{-2 \delta \rho}$ on $\left\{x \in M: \rho(x)>\frac{R(\delta)}{b}\right\}$ (with $\frac{b}{2}<\delta<a$ ), for some sufficiently large $A>0$ and $R(\delta)>0$, both dependent on $\delta$, which in turns allows us to obtain (see the proof of Proposition 4.3 for the technical details)

- the integral of $\left|\nabla\left\{|\nabla F|^{2}+A e^{-2 \delta \rho}\right\}\right|$ over any geodesic ball $B_{x}\left(3\left(1+\frac{1}{b}\right)\right)$ lying within the outer region $\left\{x \in M: \rho(x)>\frac{R(\delta)}{b}\right\}$ is bounded above by $C\left(a, b ;\|\phi\|_{\infty}\right) e^{-2 \delta \rho(x)}$.

Next, since $\left|\nabla\left\{|\nabla F|^{2}+A e^{-2 \delta \rho}\right\}\right|$ is continuous on $M$, to see the extent to which the above fact can ensure the finiteness of the integral $\int_{M}\left|\nabla\left\{|\nabla F|^{2}+A e^{-2 \delta \rho}\right\}\right|$, we just have to further decompose the outer region $\left\{x \in M: \rho(x)>\frac{R(\delta)}{b}\right\}$ into a countable number of rings $\{x \in M: k-1 \leq \rho(x) \leq k+1\}$, indexed by sufficiently large positive integers $k$; and argue, as in our covering Lemma 4.1 that, due to the lower bound $-b^{2}$ on the sectional curvature $K_{M}$, it is sufficient to use a total of $\left[\pi e^{b k}\right]+1$ geodesic balls with radius $3\left(1+\frac{1}{b}\right)$ to cover the ring $\{x \in M: k-1 \leq \rho(x) \leq k+1\}$, which in turns ensures that (see the proof of Proposition 4.3 for more details)

- the inequality $\int_{B_{x}\left(3\left(1+\frac{1}{b}\right)\right)}\left|\nabla\left\{|\nabla F|^{2}+A e^{-2 \delta \rho}\right\}\right| \leq C\left(a, b ;\|\phi\|_{\infty}\right) e^{-2 \delta \rho(x)}$ for any geodesic ball $B_{x}\left(3\left(1+\frac{1}{b}\right)\right) \subset\left\{x \in M: \rho(x)>\frac{R(\delta)}{b}\right\}$ is strong enough to deduce that the integral of $\left|\nabla\left\{|\nabla F|^{2}+A e^{-2 \delta \rho}\right\}\right|$ over the outer region $\left\{x \in M: \rho(x)>\frac{R(\delta)}{b}\right\}$ is finite (thanks to the condition $0<\frac{b}{2}<a$, which allows $\delta$ to be within the range $\frac{b}{2}<\delta<a$ ).

The above observation gives $\int_{M}\left|\nabla\left\{|\nabla F|+A e^{-2 \delta \rho}\right\}\right|<\infty$. Due to the fact that $\int_{M} e^{-2 \delta \rho}<\infty$, which is ensured by the condition $2 \delta>b$ (see inequality (4.18)), we finally conclude that $\int_{M}\left|\nabla\left\{|\nabla F|^{2}\right\}\right|<\infty$, which is a backbone ensuring the correctness of equation (1.10) .

From the above discussion, one can see that the main difficulty is to show that our solutions have finite energy and finite dissipation. We really would like to emphasize this point. For simplicity we restrict our attention to the space form $\mathbb{H}^{2}\left(-a^{2}\right)$, but the same discussion applies to the more general manifolds we consider.

The argument above gives $\int_{\mathbb{H}^{2}\left(-a^{2}\right)} \bar{g}(\bar{\nabla}(d F), \bar{\nabla}(d F))=a^{2} \int_{M}|\nabla F|^{2}$. But on the other hand, we can easily show that (see Corollary [5.2)

$$
\int_{\mathbb{H}^{2}\left(-a^{2}\right)} \bar{g}(\bar{\nabla}(d F), \bar{\nabla}(d F))=\int_{\mathbb{H}^{2}\left(-a^{2}\right)} \bar{g}(\operatorname{Def}(d F), \operatorname{Def}(d F)) .
$$

Hence, we have $\int_{\mathbb{H}^{2}\left(-a^{2}\right)} \bar{g}(\operatorname{Def}(d F), \operatorname{Def}(d F))$, which plays the role of the dissipation term, equals $a^{2} \int_{M}|\nabla F|^{2}$. 
At this time, it seems that it is very tempting to try to arrive at the equality

$$
\int_{\mathbb{H}^{2}\left(-a^{2}\right)} \bar{g}(\bar{\nabla}(d F), \bar{\nabla}(d F))=a^{2} \int_{\mathbb{H}^{2}\left(-a^{2}\right)}|\nabla F|^{2}
$$

by a more direct (but incorrect) way as follows.

- Since we have $\bar{\nabla}^{*} \bar{\nabla}=\left(d d^{*}+d^{*} d\right)$ - Ric for smooth divergence free 1-forms on $\mathbb{H}^{2}\left(-a^{2}\right)$, we have

$$
\bar{\nabla}^{*} \bar{\nabla}(d F)=\left(d d^{*}+d^{*} d\right)(d F)+a^{2} d F=a^{2} d F .
$$

If we were able to integrate by parts, then, we would immediately yield

$$
\int_{\mathbb{H}^{2}\left(-a^{2}\right)} \bar{g}(\bar{\nabla}(d F), \bar{\nabla}(d F))=\int_{\mathbb{H}^{2}\left(-a^{2}\right)} g\left(d F, \bar{\nabla}^{*} \bar{\nabla}(d F)\right)=a^{2} \int_{\mathbb{H}^{2}\left(-a^{2}\right)} g(d F, d F) .
$$

The above way to arrive at $\int_{\mathbb{H}^{2}\left(-a^{2}\right)} \bar{g}(\bar{\nabla}(d F), \bar{\nabla}(d F))=a^{2} \int_{\mathbb{H}^{2}\left(-a^{2}\right)} g(d F, d F)$ cannot be justified, since it is well known that, in the case of noncompact Riemannian mainfold, the validity of the integration by parts formula $\int_{\mathbb{H}^{2}\left(-a^{2}\right)} \bar{g}(\bar{\nabla}(d F), \bar{\nabla}(d F))=$ $\int_{\mathbb{H}^{2}\left(-a^{2}\right)} g\left(d F, \bar{\nabla}^{*} \bar{\nabla}(d F)\right)$ is not known in general (see for instance a remark by Joyce 14). Roughly speaking, the survival of the above integration by parts formula critically depends on the decay property of the term $\bar{\nabla}(d F)$, due to the fact that the decay rate of $d F$ (which is at most $e^{-\delta \rho}$ with $\delta$ to be as close to $a$ as possible) is not enough to beat down the growth rate of the circumfrence of the circle with radius $\rho$ (which is exactly $e^{a \rho}$ ). So, ideally, if one really insisted in saving the validity of the integration by parts formula, one would need to have some exponential dacay of $\bar{\nabla}(d F)$ as the radius $\rho$ tends to infinity to ensure that the exponential decay of $\bar{\nabla}(d F)$ and that of $d F$ all together could beat the growth rate of the circumference of the circle with radius $\rho$ in $\mathbb{H}^{2}\left(-a^{2}\right)$. But, such an exponential decay rate of $\bar{\nabla}(d F)$ is by no means easy to come by, since we cannot apply the gradient estimate of S.T. Yau, because $d F$ is not a harmonic function. So, the point is that, demonstrating the validity of the integration by parts formula is not easier or faster than our way of justifying (1.10) by using formula (1.9) in a clever way.

Finally, (1.10) has nothing to do with the Navier-Stokes equation, and it could also be of independent interest since it gives a nice relationship between the $L^{2}$ norm of $\bar{\nabla}(d F)$ and the $L^{2}$ norm of the gradient of the bounded harmonic function $F$.

1.4. Organization of the article. In order to make the paper as self-contained as possible in Section 2 we collect some facts from the differential geometry and in particular some background specific to the negatively curved manifolds.

Once that is done, we are ready to establish fundamental statements needed for the proof of Theorems 1.3 1.7 and their corollaries. They are:

1) Exponential decay of the gradient of bounded harmonic functions on negatively curved manifold-Section 3 .

2) Finiteness of $\left\|\nabla|\nabla F|^{2}\right\|_{L^{1}\left(\mathbb{H}^{2}\left(-a^{2}\right)\right)}$-Section 4.

3) Global energy inequality tools-Section 5.

Section 6 contains the proofs of the main results. 


\section{Acknowledgements}

The first author thanks the Department of Mathematics at University of Toronto for its hospitality during the author's visit, during which part of this work was carried out, as well as the Institute for Mathematics and its Applications, where the author was in residence.

\section{Preliminaries}

In this section we gather all the necessary tools from the literature needed in our proof. A lot of it relies on [27, and we list it here for the convenience of the reader. We also develop some precise statements regarding the volume forms on the negatively curved manifolds.

2.1. The Levi-Civita connection, deformation tensor and Ricci curvature. Here we recall some general background from Riemannian geometry (see for example [13, 23, 19]). In particular, we take a closer look at the deformation tensor mentioned in the introduction.

Let $M$ be an $n$-dimensional complete Riemannian manifold, and $T M$ and $T^{*} M$ tangent and cotangent bundles on $M$ respectively. Let $g$ be a Riemannian metric, $g(\cdot, \cdot) \in C^{\infty}\left(M, S^{2} T^{*} M\right)$, where $S^{2} T^{*} M$ denotes symmetric bilinear forms on $T M$, and $\bar{\nabla}$ the Levi-Civita connection on $(M, g)$,

$$
\bar{\nabla}: C^{\infty}(M, T M) \rightarrow C^{\infty}\left(M, T^{*} M \otimes T M\right) .
$$

Let $\langle\cdot, \cdot\rangle_{T M \otimes T^{*} M}$ be the natural pairing between vector fields and 1-forms on $M$. Given a vector field $X \in C^{\infty}(M ; T M)$, using the metric $g$ we can define $X^{*} \in$ $C^{\infty}\left(M ; T^{*} M\right)$ (lower the index) by

$$
\left\langle Y, X^{*}\right\rangle_{T M \otimes T^{*} M}=g(X, Y), \quad Y \in C^{\infty}(M ; T M) .
$$

Similarly, given a 1 -form $\omega \in C^{\infty}\left(M ; T^{*} M\right)$ we can define $v_{\omega} \in C^{\infty}(M ; T M)$ (raise the index) by

$$
g\left(v_{\omega}, Y\right)=\langle Y, \omega\rangle_{T M \otimes T^{*} M} .
$$

Therefore, the Riemannian metric $g$ gives rise to the natural identification

$$
C^{\infty}(M ; T M) \cong C^{\infty}\left(M ; T^{*} M\right)
$$

. In particular, if $F$ is a smooth function on $M$, and $d$ is the exterior derivative we have

$$
(\nabla F)^{*}=d F
$$

which is often taken as the definition of the gradient $\nabla F$. Next $g$ also induces its dual metric $g^{*}(\cdot, \cdot) \in C^{\infty}\left(M, S^{2} T M\right)$ by

$$
g^{*}(\omega, \gamma)=g\left(v_{\omega}, v_{\gamma}\right), \quad \omega, \gamma \in T^{*} M .
$$

Then note

$$
|d F|^{2}=g^{*}(d F, d F)=g(\nabla F, \nabla F)=|\nabla F|^{2} .
$$

Now by using $g$ again, we can also induce the corresponding positive definite inner product $\bar{g}(\cdot, \cdot)$ on the bundle $T^{*} M \otimes T^{*} M$, which is precisely characterized by the following condition

- Let $e_{1}, e_{2}, \ldots, e_{n}$ be a local orthonormal moving frame for $T M$, and let $\theta^{1}, \theta^{2}, \ldots \theta^{n}$ be the corresponding dual frame for $T^{*} M$, then, the list $\left\{\theta^{i} \otimes\right.$ $\left.\theta^{j}: 1 \leq j, k \leq n\right\}$ is orthonormal with respect to $\bar{g}(\cdot, \cdot)$. 
Next, the Levi-Civita connection $\bar{\nabla}$ on the tangent bundle $T M$ induces the associated Levi-Civita connection $\bar{\nabla}$ on $T^{*} M$ by

$$
\begin{aligned}
& \bar{\nabla}: C^{\infty}\left(M, T^{*} M\right) \rightarrow C^{\infty}\left(M, T^{*} M \otimes T^{*} M\right), \\
& \left(\bar{\nabla}_{X} \omega\right)(Y)=\left(\bar{\nabla}_{X} v_{\omega}\right)^{*}(Y), \quad \omega \in T^{*} M, X, Y \in T M .
\end{aligned}
$$

Notice, for the sake of convenience and keeping with the conventions, we use the same abbreviation $\bar{\nabla}$ to denote both the Levi-Civita connection on $T M$ and the induced connection on $T^{*} M$. No possible confusion should arise since the meaning of the symbol $\bar{\nabla}$ will be clear from the context. In particular, we have, by the definition of the induced connection $\bar{\nabla}$ on $T^{*} M$, the property that

$$
\bar{\nabla}_{X} Y^{*}=\left(\bar{\nabla}_{X} Y\right)^{*}
$$

for any smooth vector fields $X, Y$ on $M$.

We now turn our attention to the deformation tensor

$$
\text { Def : } C^{\infty}(M, T M) \rightarrow C^{\infty}\left(M, S^{2} T^{*} M\right),
$$

defined by (see for example [4])

$$
(\operatorname{Def} X)(Y, Z)=\frac{1}{2}\left\{g\left(\bar{\nabla}_{Y} X, Z\right)+g\left(\bar{\nabla}_{Z} X, Y\right)\right\}, \quad Y, Z \in C^{\infty}(M, T M) .
$$

Using the natural identification of the space of vector fields with the space of 1forms on $M$ discussed above, the operator Def can be regarded as the operator from $C^{\infty}\left(M, T^{*} M\right)$ to $C^{\infty}\left(M, S^{2} T^{*} M\right)$, and can be defined alternatively as follows:

Definition 2.1. For any 1-form $\omega \in C^{\infty}\left(M, T^{*} M\right)$, the deformation tensor Def $\omega \in C^{\infty}\left(M, S^{2} T^{*} M\right)$ is given by

$$
(\operatorname{Def} \omega)(Y, Z)=\frac{1}{2}\left\{\left\langle Z, \bar{\nabla}_{Y} \omega\right\rangle_{T M \otimes T^{*} M}+\left\langle Y, \bar{\nabla}_{Z} \omega\right\rangle_{T M \otimes T^{*} M}\right\},
$$

for any $Y, Z \in C^{\infty}(M, T M)$.

In the sequel we also need the following. If we express $\omega \in C^{\infty}\left(M, T^{*} M\right)$ locally as $\omega=\sum_{a} \omega_{a} d x^{a}$, then Def $\omega$ can locally be expressed as

$$
\operatorname{Def} \omega=\sum_{j, k} \frac{1}{2}\left(\omega_{j ; k}+\omega_{k ; j}\right) d x^{j} \otimes d x^{k},
$$

where $\omega_{j ; k}=\partial_{k} \omega_{j}-\Gamma_{j k}^{l} \omega_{l}$, where $\Gamma_{j k}^{l}$ are the Christoffel symbols.

Next we discuss the Ricci curvature on a complete $n$-dimensional Riemannian manifold $M$. Recall, the Ricci curvature is a symmetric tensor Ric $\in C^{\infty}\left(M, S^{2} T^{*} M\right)$ defined as follows

$$
\operatorname{Ric}_{p}(X, X)=\sum_{1 \leq i \leq n-1} K_{M}\left(X, e_{i}\right), \quad p \in M
$$

where $e_{1}, e_{2}, \ldots e_{n-1}$ are some unit vectors in $T_{p} M$ such that $\left\{X, e_{1}, \ldots e_{n-1}\right\}$ forms an orthonormal basis for $T_{p} M$. In many occasions, we use the symbol $\operatorname{Ric}(M)$ for Ric. Moreover, if we write $\operatorname{Ric}(M) \geq-b^{2}$, it means that $\operatorname{Ric}(X, X) \geq-b^{2}|X|^{2}$, for all $X \in C^{\infty}(M, T M)$. Moreover, it is clear that, for 2-dimensional Riemannian manifold $M$, the notion of Ricci curvature Ric coincides with the sectional curvature $K_{M}$.

Besides the Ricci curvature tensor Ric $\in C^{\infty}\left(M, T^{*} M \otimes T^{*} M\right)$, we also need 
to consider the Ricci operator Ric : $C^{\infty}\left(M, T^{*} M\right) \rightarrow C^{\infty}\left(M, T^{*} M\right)$ sending the space of 1-forms into itself. It is defined by

$$
\operatorname{Ric}\left(u^{*}\right)=\sum_{a, b} \eta^{a} g\left(R\left(e_{a}, e_{b}\right)\left(e_{b}\right), u\right)
$$

where $R(\cdot, \cdot)$ is the Riemannian curvature tensor, $e_{1}, e_{2}, \ldots e_{n}$ is a local orthonormal moving frame for $T M$, and $\eta^{1}, \eta^{2}, \ldots \eta^{n}$ stand for the associated dual frame for $T^{*} M$ with respect to $e_{1}, e_{2}, \ldots e_{n}$.

In the case of the space form $\mathbb{H}^{n}\left(-a^{2}\right)$ with sectional curvature $-a^{2}$, we have $R\left(e_{a}, e_{b}\right)\left(e_{b}\right)=-a^{2} e_{a}$, for any local orthonormal frame $e_{1}, e_{2}, \ldots e_{n}$ of $T \mathbb{H}^{n}\left(-a^{2}\right)$. Hence, in particular we have the following fact

$$
\begin{aligned}
\operatorname{Ric}\left(u^{*}\right) & =\sum_{a, b} \eta^{a} g\left(R\left(e_{a}, e_{b}\right)\left(e_{b}\right), u\right) \\
& =-a^{2}(n-1) \sum_{a} \eta^{a} g\left(e_{a}, u\right) \\
& =-a^{2}(n-1) \sum_{a} u^{a} \eta^{a} \\
& =-a^{2}(n-1) u^{*}
\end{aligned}
$$

We end this section with a quick summary of the facts we use in sequel:

$$
\begin{aligned}
\operatorname{Ric}_{p}(X, X) & =K_{M}(p)|X|^{2}, \quad p \in M, X \in C^{\infty}(M, T M), \operatorname{dim} M=2, \\
(2.12) \quad \operatorname{Ric}(\omega) & =-(n-1) a^{2} \omega, \quad \omega \in C^{\infty}\left(H^{n}\left(-a^{2}\right), T^{*} H^{n}\left(-a^{2}\right)\right), \quad a>0, n \geq 2 .
\end{aligned}
$$

2.2. Estimates and identities used. As usual, we start with a complete $n$ dimensional Riemannian manifold $M$, and consider the geodesic normal coordinates on $M$ about a selected base point $O$. One of the fundamental properties of the normal coordinates, which we use in computations, is that the Christoffel symbols all vanish at $O$ :

$$
\Gamma_{j k}^{i}=0
$$

(see for example [19]).

In the case of a complete, simply connected, $n$-dimensional Riemannian manifold $M$ with sectional curvature $-b^{2} \leq K_{M} \leq a^{2}<0$, the Cartan-Hadamard theorem ensures that the geodesic normal coordinates on $M$ about any selected base point $O \in M$ must be globally defined, which also implies in particular that $M$ is diffeomorphic to $\mathbb{R}^{n}$. Moreover, in this case, between any two points $p, q$ the geodesic joining $p$ and $q$ is unique, and hence we just use the symbols $\overline{p q},|\overline{p q}|$ to denote the geodesic joining $p$ and $q$, and its length respectively.

Define the distance function from a point $p \in M$ to a point $x$ by

$$
\rho_{p}(x) \equiv|\overline{p x}| \text {. }
$$

We usually omit the subscript $p$ and simply write $\rho(x)$ since the base point is clear from the context. 
Lemma 2.2 (Properties of the distance function [27, Ch. 1]). The distance function $\rho(x)$ defined as above is smoot 12 on all of $M$, where $M$ is any Riemannian $n$-manifold such that the exponential map defines the diffeomorphism between $M$ and $\mathbb{R}^{n}$. In addition,

$$
\begin{aligned}
|\nabla \rho|^{2} & =1, \\
\Delta \rho & =(n-1) k \operatorname{coth} k \rho \quad \text { if } M \text { has constant sectional curvature }=-k^{2}, \\
\Delta \rho & \geq(n-1) a \cdot \operatorname{coth}(a \rho) \geq(n-1) a \quad \text { if } \operatorname{Ric}(M) \leq-a^{2}, \\
\Delta \rho & \leq(n-1) b \cdot \operatorname{coth}(b \rho) \leq(n-1) \frac{1+b \rho}{\rho} \quad \text { if } \operatorname{Ric}(M) \geq-(n-1) b^{2} .
\end{aligned}
$$

Lemma 2.3. 27. p.35] Let $M$ be a simply connected, $n$-dimensional, complete Riemannian manifold with sectional curvature satisfying $-b^{2} \leq K_{M} \leq-a^{2}$. Let $O \in M$ be a selected base point, and let $x_{1}, x_{2}$ be two points in $M$ for which $\left|\overline{O x_{1}}\right|=\left|\overline{O x_{2}}\right|=R$. Moreover, let $\theta=\angle\left(\overline{O x_{1}}, \overline{O x_{2}}\right)$. Then there exist universal constants $\tilde{R}_{0}$ (depending only on $a, b$, and $n$ ), and $\theta_{0} \in\left(0, \frac{\pi}{2}\right)$, such that whenever $\left|\overline{O x_{1}}\right|=\left|\overline{O x_{2}}\right|=R \geq \tilde{R}_{0}$, and $\theta \leq \theta_{0}$, we have the following

$$
2 R+\frac{2}{a}(\log \theta-1) \leq\left|\overline{x_{1} x_{2}}\right| \leq 2 R+\frac{2}{b}(\log \theta+1) .
$$

Lemma 2.4. With $M$ as above, let $O \in M$ be a selected base point, and let $x_{1}, x_{2}$ be two points in $M$ for which $\left|\overline{O x_{1}}\right|=\left|\overline{O x_{2}}\right|=R$. In addition require $\left|\overline{x_{1} x_{2}}\right| \leq 1$. Then there exist $R_{1}$ (depending only on a) such that if $R \geq R_{1}$, then $\theta=\angle\left(\overline{O x_{1}}, \overline{O x_{2}}\right) \leq \theta_{0}$ from Lemma 2.3.

Proof. Using the intuition from the Euclidean geometry it is very easy to see what this lemma means: if we go far enough from the origin and the points $x$ and $y$ are close to one another, the polar angle between them has to be small enough. To prove this for negatively curved manifolds we use the following inequality from 27. (1.9) p.34]

$$
\cos \theta \geq \operatorname{coth}^{2}(a R)-\frac{\cosh \left(a\left|x_{1} x_{2}\right|\right)}{\sinh ^{2}(a R)},
$$

where $x_{1}, x_{2} \in M, R=\left|O x_{1}\right|=\left|O x_{2}\right|$, and $\theta=\angle\left(\overline{O x_{1}}, \overline{O x_{2}}\right)$. Now, if $\left|x_{1} x_{2}\right| \leq 1$, then

$$
\cos \theta \geq \operatorname{coth}^{2}(a R)-\frac{\cosh (a)}{\sinh ^{2}(a R)} .
$$

Since the right hand side goes to 1 as $R \rightarrow \infty$, there must exist $R_{1}$ such that if $R \geq R_{1}$, then

$$
\operatorname{coth}^{2}(a R)-\frac{\cosh (a)}{\sinh ^{2}(a R)}>\cos \theta_{0} .
$$

This means that if $R \geq R_{1}$, we must have $\theta \leq \theta_{0}$. Otherwise the monotonicity of $\cos \theta$ on $\left(0, \frac{\pi}{2}\right)$ and (2.19) would imply

$$
\cos \theta_{0}>\cos \theta \geq \operatorname{coth}^{2}(a R)-\frac{\cosh (a)}{\sinh ^{2}(a R)}>\cos \theta_{0} .
$$

\footnotetext{
${ }^{2}$ For more general manifolds $M, \rho(x)$ is smooth on $M \backslash C u t(p)$. See 27 for precise definitions and statements.
} 
LEMMA 2.5. 27. p.78] Let $M$ be a general $n$-dimensional complete Riemannian manifold. Suppose that $f: B((1+\tau) R) \rightarrow[0, \infty)$ is a non-negative subharmonic function on $B((1+\tau) R)$ (i.e., $f \geq 0$ and $\Delta f \geq 0$ on $B((1+\tau) R))$. Then

$$
\int_{B(R)}|\nabla f|^{2} \leq \frac{C}{\tau^{2} R^{2}} \int_{B((1+\tau) R)} f^{2},
$$

where $C$ is some universal constant.

We now give the statement of the gradient estimate of Yau.

Theorem 2.6 (Gradient Estimate [34, 27]). Let $M$ be an n-dimensional complete Riemannian manifold with $\operatorname{Ric}(M) \geq-(n-1) K$, for some constant $K \geq 0$. If $u$ is a positive harmonic function on $M$ and $B_{r}(x)$ is a geodesic ball in $M$, then

$$
|\nabla u| \leq C_{n}\left(\frac{1+r \sqrt{K}}{r}\right) u \quad \text { on } B \frac{r}{2}(x),
$$

where $C_{n}$ is a constant depending only on $n$.

We finish the section by stating the important formula mentioned in the introduction.

LEMMA 2.7. 27 p. 15] Let $M$ be a Riemannian manifold. Then the following holds in normal coordinates at $x$

$$
\Delta\left[|\nabla F|^{2}\right](x)=2 \sum\left[\partial_{i} \partial_{j} F\right]^{2}(x)+2 \sum \partial_{i} F(x) \partial_{i}(\Delta F)(x)+2 \operatorname{Ric}(\nabla F, \nabla F)(x) .
$$

2.3. The volume form and the comparison theorem for Jacobi fields. In this section we derive the geodesic (normal) polar coordinates. The reader can choose to skip to equation (2.26) and to the Theorem [2.8 and the remarks afterwards.

We consider a complete, simply connected, 2-dimensional Riemannian manifold $M$ with sectional curvature satisfying $-b^{2} \leq K_{M} \leq-a^{2}<0$. To begin, let $O$ be a selected point in $M$, and let $\exp _{O}: T_{O} M \rightarrow M$ be the global exponential map at $O$, whose existence is ensured as before by the Cartan-Hadamard theorem. We remark that the tangent space $T_{O} M$ is identified with the Euclidean space $\mathbb{R}^{2}$.

Let $(\bar{r}, \bar{\theta})$ be the ordinary polar coordinates on $\mathbb{R}^{2}$ in the Euclidean sense. That is, the respective induced vectors $\frac{\partial}{\partial \bar{r}}$ and $\frac{\partial}{\partial \bar{\theta}}$ are given by

$$
\begin{aligned}
& \left.\frac{\partial}{\partial \bar{r}}\right|_{(x, y)}=\frac{(x, y)}{\left(x^{2}+y^{2}\right)^{\frac{1}{2}}}, \\
& \left.\frac{\partial}{\partial \bar{\theta}}\right|_{(x, y)}=(-y, x) .
\end{aligned}
$$

Then, the geodesic normal polar coordinates $(r, \theta)$ on $M$ (as induced by $\exp _{O}$ : $\left.T_{O} M \rightarrow M\right)$ is given by the composite function $(r, \theta)=(\bar{r}, \bar{\theta}) \circ\left\{\exp _{O}\right\}^{-1}$.

Let $v \in T_{O} M=\mathbb{R}^{2}$ be any unit vector, and consider the geodesic $c:[0, \infty) \rightarrow$ $M$ with $c(0)=O$ and $c^{\prime}(0)=v$. Then, we notice that $\frac{\partial}{\partial r}{ }_{c(r)}=c^{\prime}(r)$. Next, in order to compute $\frac{\partial}{\partial \theta} c(r)$, we first observe that the ordinary Euclidean $\frac{\partial}{\partial \bar{\theta}} r v$ is given by

- $\frac{\partial}{\partial \bar{\theta}} r v=r w$, in which $w$ is the unique unit vector in $\mathbb{R}^{2}$ such that the pair $\{v, w\}$ forms a positively oriented orthonormal basis for $\mathbb{R}^{2}$. (Recall that 
we have the identification $T_{O} M=\mathbb{R}^{2}$, so, we may just regard $v \in T_{O} M$ to be a vector in $\mathbb{R}^{2}$.)

Now, let $Y(r)$ be the Jacobi field along the geodesic $c:[0, \infty) \rightarrow M$ with $Y(0)=0$, and $\nabla_{\frac{\partial}{\partial r}} Y(0)=w$. (Recall that $Y(r)$ is a Jacobi field means that the equation $\bar{\nabla}_{c^{\prime}} \bar{\nabla}_{c^{\prime}} Y+R\left(Y, c^{\prime}\right) c^{\prime}=0$ holds along the geodesic ray $c(t)$.) Then, it is well known that 13

$$
Y(r)=\left(D \exp _{O}\right)_{r v}(r w)=\left(D \exp _{O}\right)_{r v}\left(\frac{\partial}{\partial \bar{\theta}_{r v}}\right),
$$

which implies that $\frac{\partial}{\partial \bar{\theta}} r v=\left(D \exp _{O}^{-1}\right)_{c(r)}(Y(r))$. Hence, we have for any $f \in C^{\infty}(M)$

$$
\begin{aligned}
\frac{\partial}{\partial \theta}_{c(r)} f & =\frac{\partial}{\partial \bar{\theta}_{r v}}\left(f \circ \exp _{O}\right) \\
& =\left[\left(D \exp _{O}^{-1}\right)_{c(r)}(Y(r))\right]\left(f \circ \exp _{O}\right)=d\left(f \circ \exp _{O}\right)_{r v}\left(\left(D \exp _{O}^{-1}\right)_{c(r)}(Y(r))\right) \\
& =(d f)_{c(r)}(Y(r))=Y(r)(f) . \\
& \frac{\partial}{\partial \theta}_{c(r)}=Y(r) .
\end{aligned}
$$

With the above preparation, we can now discuss the growth rate of the volume form on a complete, simply connected, 2-dimensional Riemannian manifold with $-b^{2} \leq K_{M} \leq-a^{2}<0$. Under the geodesic normal coordinates $(r, \theta)$, the volume form is given by

$$
\left|\frac{\partial}{\partial \theta}\right| d r d \theta
$$

but sometimes we write $\left|\frac{\partial}{\partial \theta}\right| d \rho d \theta$ in the case when the distance function $\rho$ from $O$ is used to replace the symbol $r$. Then, the following comparison theorem in differential geometry is used to give us the growth rate of the volume form.

Theorem 2.8. [Comparison theorem for Jacobi fields 13 . Corollary 4.6.1, p.209]] Let $M$ be a simply connected, complete, $n$-dim Riemannian manifold $M$ with $-b^{2} \leq K_{M} \leq-a^{2}<0$. Let $c:[0, \infty) \rightarrow M$ be a geodesic ray in $M$, and we consider a given Jacobi field $Y(r)$ along the geodesic ray $c$ which is orthogonal to $c$ and satisfies $Y(0)=0$ and $|\dot{Y}(0)|=1$. Then

$$
\frac{1}{a} \sinh (a r) \leq|Y(r)| \leq \frac{1}{b} \sinh (b r) .
$$

Now, by the above comparison theorem together with the identity $\frac{\partial}{\partial \theta} c(r)=$ $Y(r)$, we immediately deduce that, for a complete, simply connected Riemannian manifold $M$ of dimension 2 with $-b^{2} \leq K_{M} \leq-a^{2}<0$, the weight $G(r, \theta)=\left|\frac{\partial}{\partial \theta}\right|$ of the volume form $\left|\frac{\partial}{\partial \theta}\right| d r d \theta$ on $M$ is at most of the order $\frac{1}{b} \sinh (b r)$. This is used in Sections 3 and 4 to estimate the integrals of a certain non-negative function on $M$. Finally, we also remark that, in the special case of a space form $\mathbb{H}^{2}\left(-a^{2}\right)$, we have exactly

$$
\left|\frac{\partial}{\partial \theta}\right|=|Y(r)|=\frac{1}{a} \sinh (a r)
$$


2.4. Geodesic balls, cones and the geometric boundary $S(\infty)$. We again only consider simply connected, complete, $n$-dimensional Riemannian manifold $M$ with sectional curvature $-b^{2} \leq K_{M} \leq-a^{2}<0$.

Using the distance function from the Section 2.2 define a geodesic ball in $M$ with radius $R$ and centered at $x$ by

$$
B_{R}(x)=\left\{y \in M: \rho_{x}(y) \leq R\right\} .
$$

Next, let $O \in M$ and $v \in T_{O} M$. Define the cone about $v$ with angle $\theta$ by

$$
C_{O}(v, \theta)=\{y \in M: \angle(v, \overline{O y}) \leq \theta\} .
$$

Finally the geometric boundary, the sphere at infinity $S(\infty)$ is

$$
S(\infty)=\text { the set of all geodesic rays from } O,
$$

which can be canonically identified with the unit sphere in $T_{O} M: S_{O}(1)=\{v \in$ $\left.T_{O} M:|v|=1\right\}$, so that every unit vector $v \in T_{O} M$ can then be regarded as a point in $S(\infty)$, and we can simply write $v \in S(\infty)$ (See [27, p.36], [1, 2] ).

From now on

$$
\bar{M}=M \cup S(\infty) .
$$

2.5. Bounded harmonic functions on negatively curved manifolds. Anderson [1] and Sullivan [29] independently, and using different methods, proved the following theorem.

TheOREm 2.9. 10, 29 Let $M$ be a simply connected, n-dimensional, complete Riemannian manifold with sectional curvature $K_{M}$ satisfying $-b^{2} \leq K_{M} \leq-a^{2}<$ 0 . Then there exists a unique solution $u \in C^{\infty}(M) \cap C^{0}(\bar{M})$ to the Dirichlet problem

$$
\begin{aligned}
& \Delta u=0 \text { in } M, \\
& \left.u\right|_{S(\infty)}=\phi \in C^{0}(S(\infty)) .
\end{aligned}
$$

A simpler proof is also presented in the comprehensive work of Anderson and Schoen [2] and it is also exposed in [27. The main idea there is to construct two barrier functions and use the Perron's method 3 . This in turn is accomplished in three steps, whose conclusions we use in the proof of Proposition 3.1 which is a crucial tool for our result. Therefore, we give an outline of the proof in [27. p. 37], and list the needed conclusions:

Step 0) Due to the classical elliptic theory, the proof can assume $\phi \in C^{\infty}(S(\infty))$, but we emphasize that for the existence of $F$ it is enough to assume $\phi$ is only continuous. On the other hand, when we use the steps below, we will require $\phi \in C^{1}(S(\infty))$.

Step 1) Extend the function $\phi$ to all of $M$ and show for $x \in M$

$$
\underset{B_{x}(1)}{\operatorname{osc}} \phi \equiv \sup _{y \in B_{x}(1)}|\phi(y)-\phi(x)| \leq C_{0} e^{-a \rho(x)},
$$

where $C_{0}$ can be shown to be given by

$$
C_{0}=\max \left\{e^{\frac{a}{2}+1}\left\|\phi^{\prime}\right\|_{L^{\infty}(S(\infty))}, 2 e^{a R_{1}}\|\phi\|_{L^{\infty}(S(\infty))}\right\},
$$

and $R_{1}$ is obtained using Lemma 2.4

\footnotetext{
${ }^{3}$ We note that the proof in $\mathbf{1}$ also relies on the Perron's method.
} 
To extend $\phi$ to all of $M$, pick a base point $O \in M$ and use the geodesic normal polar coordinates $(r, \theta)$ at $O$ to define

$$
\phi(r, \theta)=\phi(\theta), \text { for all } r>0 .
$$

Step 2) The Laplacian of the average of $\phi$ has an exponential decay. More precisely, let

$$
\bar{\phi}(x)=\frac{\int \chi\left(\rho_{x}^{2}(y)\right) \phi(y) d y}{\int \chi\left(\rho_{x}^{2}(y)\right) d y},
$$

where $\chi$ is a standard cut off function. Then it can be shown

$$
\begin{aligned}
\underset{B_{x}(1)}{\operatorname{osc}} \bar{\phi} & \leq C e^{-a \rho(x),}, \\
|\Delta \bar{\phi}(x)| & \leq C e^{-a \rho(x)},
\end{aligned}
$$

where $\rho(x)$ is the distance function defined in Section 2.2. We note the above constant is a generic constant $C$ depending on $C_{0}$ given by 2.30) and the manifold $M$.

Step 3) Show there exists $\alpha>0$ and $\delta$ small enough such that

$$
\Delta\left[\bar{\phi}-\alpha e^{-\delta \rho}\right] \geq 0 \quad \text { and } \quad \Delta\left[\bar{\phi}+\alpha e^{-\delta \rho}\right] \leq 0 .
$$

Then by Perron' 4 method there exists a harmonic function $F$ such that

$$
\bar{\phi}-\alpha e^{-\delta \rho} \leq F \leq \bar{\phi}+\alpha e^{-\delta \rho} .
$$

The boundary conditions are easily checked.

2.5.1. Constants $\alpha$ and $\delta$. Constants $\alpha$ and $\delta$ from Step 3 play a very important role in our proofs. Therefore we take some time now to discuss $\alpha$ and $\delta$, and how they relate to the function $\phi$ and the curvature $-b^{2} \leq K_{M} \leq-a^{2}$. We emphasize that this exposition is completely based on [27, p. 40] although the details of (2.37) and (2.38) below were not exposed there.

First start with some $\delta>0$ to be specified later. Using (2.14) we then observe

$$
\Delta\left(e^{-\delta \rho(x)}\right)=\delta e^{-\delta \rho(x)}(\delta-\Delta \rho(x)) .
$$

Also, by (2.16)

$$
\Delta \rho \geq(n-1) a \cdot \operatorname{coth}(a \rho) \geq(n-1) a .
$$

Next, one has to choose sufficiently small $\delta$ and sufficiently large $\alpha$ so that (2.34) does indeed hold. Let $\delta<a$, then the first equation (2.34) is obtained as follows. By (2.33) and (2.36)

$$
\begin{aligned}
\Delta\left[\bar{\phi}-\alpha e^{-\delta \rho}\right] & =\Delta \bar{\phi}-\alpha \delta e^{-\delta \rho}(\delta-\Delta \rho) \\
& \geq-C e^{-a \rho}+\alpha \delta[(n-1) a-\delta] e^{-\delta \rho} \\
& \geq\{\alpha \delta[(n-1) a-\delta]-C\} e^{-\delta \rho},
\end{aligned}
$$

where $\delta<a$ is used to obtain the last line. Similarly

$$
\begin{aligned}
\Delta\left[\bar{\phi}+\alpha e^{-\delta \rho}\right] & \leq C e^{-a \rho}-\alpha \delta[(n-1) a-\delta] e^{-\delta \rho} \\
& \leq\{C-\alpha \delta[(n-1) a-\delta]\} e^{-\delta \rho} .
\end{aligned}
$$

So, for any $\delta<a$, we choose $\alpha=\frac{2 C}{\delta[(n-1) a-\delta]}$. Note, in order to guarantee $\alpha>0$ for $n=2$, we need $\delta<a$ and not just $\delta \leq a$. Then (2.34) follows as needed.

\footnotetext{
${ }^{4}$ See 1 for the application in this context or $\mathbf{7}$.
} 
In addition, besides $\delta$ not being too large, we eventually need $\delta$ not to be too small. More precisely, when we want to obtain that $\nabla F$ is in $L^{2}\left(\mathbb{H}^{2}\left(-a^{2}\right)\right)$, we impose additional condition $\frac{a}{2}<\delta$. Then the exponential decay of $\nabla F$ obtained in Proposition 3.1 below will be sufficient to give $\|\nabla F\|_{L^{2}\left(\mathbb{H}^{2}\left(-a^{2}\right)\right)}<\infty$.

Similarly, when we want to obtain that $\nabla F$ is in $L^{2}(M)$, where $M$ is a complete, simply connected 2-dimensional manifold with sectional curvature satisfying $-b^{2} \leq$ $K_{M} \leq-a^{2}$, where $a, b>0$ and $\frac{b}{2}<a$, then there we require $\delta>\frac{b}{2}$.

2.6. Hodge Star operator and Hodge Laplacian. Let $d$ denote the exterior differentiation operator, which sends $k$ forms to $k+1$ forms. As is well-known, $d$ satisfies

$$
d d \omega=0 \quad \text { for any } k-\text { form } \omega .
$$

Its dual operator, $d^{*}$, is given by

$$
d^{*}=(-1)^{k} * * * d *,
$$

where $*$ is the Hodge $*$ operator and $k$ comes from $d^{*}$ acting on some given $k$-form (see for example [26]). We note $d^{*}$ sends $k$ forms to $k-1$ forms. However, the only main facts that we need to know from Hodge theory besides (2.39), is the definition of the Hodge Laplacian and equation (2.42) below. The Hodge Laplacian is given by

$$
-\Delta \omega=\left(d d^{*}+d^{*} d\right) \omega
$$

When $\Delta$ acts on a function $F$, then the expression simplifies to

$$
-\Delta F=d^{*} d F \text {. }
$$

So for example if we have a function $F$ that is harmonic, and if we define a 1-form $U$ by

$$
U=d F,
$$

then it is very easy to see that $U$ is a harmonic 1 -form since

$$
\begin{array}{rlr}
\left(d d^{*}+d^{*} d\right) U & =d d^{*} d F+d^{*} d d F \\
& =d d^{*} d F & \\
& =d\left(d^{*} d F\right) \\
& =0,
\end{array}
$$

where in the last line we used the fact that $F$ is harmonic and (2.41). The construction of our non-unique solution relies on this simple observation.

\section{Exponential decay of the gradient of bounded harmonic functions}

The main result of this section is the following proposition.

Proposition 3.1. Let $M$ be an $n$-dimensional complete, simply connected Riemannian manifold with sectional curvature satisfying $-b^{2} \leq K_{M} \leq-a^{2}$. Let $\phi \in C^{1}(S(\infty))$ be any boundary data, and $F \in C^{\infty}(M) \cap C^{0}(\bar{M})$ be the unique bounded harmonic function on $M$ with $\left.F\right|_{S(\infty)}=\phi$. Let $\delta<a$. Then, the following inequality holds

$$
|\nabla F|(x) \leq C\left\{1+\frac{1}{\delta[(n-1) a-\delta]}\right\} e^{-\delta \rho(x)} \quad \forall x \in M,
$$

where $C$ depends only on $a, b$, and $n$ and $C_{0}$ given by (2.30). 
Proof. Given $\phi \in C^{1}(S(\infty))$ by Theorem 2.9 there exists a unique harmonic function $F \in C^{\infty}(M) \cap C^{0}(\bar{M})$ with $\left.F\right|_{S(\infty)}=\phi$. By (2.35) we also have

$$
\bar{\phi}-\alpha e^{-\delta \rho} \leq F \leq \bar{\phi}+\alpha e^{-\delta \rho},
$$

where $\bar{\phi}$ is as in 2.33) and $\delta<a$ and $\alpha=\frac{2 C}{\delta[(n-1) a-\delta]}$ as discussed in Section 2.5.1 Let $x \in M$ and consider two cases.

Case 1: $\rho(x)>1$.

Consider a ball $B_{x}(1)$. By (3.2)

$$
\begin{aligned}
\underset{B_{x}(1)}{\operatorname{osc}} F & :=\sup _{B_{x}(1)} F-\inf _{B_{x}(1)} F \\
& \leq \sup _{B_{x}(1)}\left(\bar{\phi}+\alpha e^{-\delta \rho}\right)-\inf _{B_{x}(1)}\left(\bar{\phi}-\alpha e^{-\delta \rho}\right) .
\end{aligned}
$$

Next since $\inf _{B_{x}(1)}\left[\bar{\phi}-\alpha e^{-\delta \rho}\right] \geq \inf _{B_{x}(1)} \bar{\phi}-\alpha \cdot \sup _{B_{x}(1)} e^{-\delta \rho}$ and $\sup _{B_{x}(1)} e^{-\delta \rho}=$ $e^{-\delta(\rho(x)-1)}$, it follows that

$$
\underset{B_{x}(1)}{\operatorname{osc}} F \leq \underset{B_{x}(1)}{\operatorname{osc}} \bar{\phi}+2 \alpha e^{-\delta(\rho(x)-1)}
$$

and it implies

$$
0 \leq F-\inf _{B_{x}(1)} F \leq \underset{B_{x}(1)}{\operatorname{osc}} F \leq \operatorname{osc}_{B_{x}(1)}^{\operatorname{og}} \bar{\phi}+2 \alpha e^{-\delta(\rho(x)-1)} .
$$

Now, notice that since $F-\inf _{B_{x}(1)} F$ is a positive harmonic function on $B_{x}(1)$ we can apply the gradient estimate, Theorem 2.6 and (2.32) to deduce the following inequality for any $y \in B_{x}\left(\frac{1}{2}\right)$

$$
\begin{aligned}
|\nabla F|(y)=\left|\nabla\left[F(y)-\inf _{B_{x}(1)} F\right]\right| & \leq C(1+a)\left[F(y)-\inf _{B_{x}(1)} F\right] \\
& \leq C\left\{\underset{B_{x}(1)}{\operatorname{osc}} \bar{\phi}+2 \alpha e^{-\delta(\rho(x)-1)}\right\} \\
& \leq C\left[C+2 \alpha e^{\delta}\right] e^{-\delta \rho(x)} \\
& =C\left\{C+\frac{4 C}{\delta[(n-1) a-\delta]} e^{\delta}\right\} e^{-\delta \rho(x)} .
\end{aligned}
$$

So, in particular, if we choose $y=x$ in the above inequality, we have

$$
|\nabla F|(x) \leq C\left\{C+\frac{4 C}{\delta[(n-1) a-\delta]} e^{\delta}\right\} e^{-\delta \rho(x)} \quad \forall x \in M-B_{o}(1) .
$$

We now consider the case of $x \in B_{0}(1)$.

Case 2: $\rho(x) \leq 1$.

Here we have $e^{-a} \leq e^{-a \rho(x)}$, and $\sup _{B_{x}(1)} e^{-\delta \rho}=1$. Hence

$$
\begin{aligned}
\underset{B_{x}(1)}{\operatorname{osc} F} & \leq \underset{B_{x}(1)}{\operatorname{osc}} \bar{\phi}+2 \alpha \sup _{B_{x}(1)} e^{-\delta \rho} \\
& \leq C e^{-a \rho}+2 \alpha \\
& =C e^{-a \rho}+2 \alpha e^{a} e^{-a} \\
& \leq\left[C+2 \alpha e^{a}\right] e^{-a \rho} \\
& \leq\left[C+2 \alpha e^{a}\right] e^{-\delta \rho} \\
& =\left\{C+\frac{4 C}{\delta[(n-1) a-\delta]} e^{a}\right\} e^{-\delta \rho} .
\end{aligned}
$$


Next, as in Case 1 we can apply the gradient estimate, Theorem [2.6. to $F$ $\inf _{B_{x}(1)} F$ to obtain for any $y \in B_{x}\left(\frac{1}{2}\right)$

$$
\begin{aligned}
|\nabla F|(y)=\left|\nabla\left[F(y)-\inf _{B_{x}(1)} F\right]\right| & \leq C(1+a)\left[F(y)-\inf _{B_{x}(1)} F\right] \\
& \leq C \underset{B_{x}(1)}{\operatorname{osc}} F \\
& \leq C\left\{C+\frac{4 C}{\delta[(n-1) a-\delta]} e^{a}\right\} e^{-\delta \rho} .
\end{aligned}
$$

By taking $y=x$ in the above inequality, we deduce

$$
|\nabla F|(x) \leq C\left\{C\left\|\phi^{\prime}\right\|_{\infty}+\frac{4 C}{\delta[(n-1) a-\delta]} e^{a}\right\} e^{-\delta \rho(x)} \quad \forall x \in B_{O}(1) .
$$

By combining (3.4) and (3.6) we have that (3.1) holds for all $x \in M$ as needed.

By (3.1) and the discussion in Section 2.3 together with (2.27) and (2.28), we immediately have the following corollaries

Corollary 3.2. In addition if $\delta>\frac{a}{2}$, then

$$
\|\nabla F\|_{L^{2}\left(\mathbb{H}^{2}\left(-a^{2}\right)\right)}<\infty .
$$

Proof. There is nothing to prove. From (2.26) and (2.28) we have

$$
\|\nabla F\|_{L^{2}\left(\mathbb{H}^{2}\left(-a^{2}\right)\right)}^{2} \leq C \int_{0}^{\infty}|\nabla F|^{2} \sinh (a \rho) d \rho \leq C \int_{0}^{\infty} e^{a \rho-2 \delta \rho} d \rho<\infty .
$$

Corollary 3.3. Let $M$ be a complete, simply connected 2-dimensional manifold with sectional curvature satisfying $-b^{2} \leq K_{M} \leq-a^{2}$, where $a, b>0$ and $\frac{b}{2}<a$, if $\frac{b}{2}<\delta<a$, then

$$
\|\nabla F\|_{L^{2}(M)}<\infty \text {. }
$$

Proof. Similarly by (2.26) and (2.27)

$$
\|\nabla F\|_{L^{2}(M)}^{2} \leq C \int_{0}^{\infty}|\nabla F|^{2} \sinh (b \rho) d \rho \leq C \int_{0}^{\infty} e^{b \rho-2 \delta \rho} d \rho<\infty .
$$

\section{The proof that $\left\|\nabla|\nabla F|^{2}\right\|_{L^{1}\left(\mathbb{H}^{2}\left(-a^{2}\right)\right)}$ is finite}

In the proof of $\left\|\nabla|\nabla F|^{2}\right\|_{L^{1}\left(\mathbb{H}^{2}\left(-a^{2}\right)\right)}<\infty$, we need the assistance of the following geometric lemma, which is itself a consequence of Lemma 2.3 and Lemma 2.4

Lemma 4.1. [Covering Lemma] Consider $M$ to be a simply connected, complete 2-dimensional Riemannian manifold with sectional curvature $-b^{2} \leq K_{M} \leq-a^{2}$. Let $O$ be a selected base point in $M$, and let $\rho$ be the distance function from $O$. Then, there exists some sufficiently large universal constant $\bar{R}_{0}>0$ such that the following assertion holds:

For any given $R \geq \bar{R}_{0}$, if we take the positive integer $N(R)=\left[\frac{2 \pi}{2 e^{-b R}}\right]+1=$ $\left[\pi e^{b R}\right]+1$ (here, the symbol $[\lambda]$ means the largest integer $N \in \mathbb{Z}$ with $N \leq \lambda$ ), 
then, we can pick a list of vectors $v_{1}, v_{2}, v_{3}, \ldots . v_{N(R)} \in S(\infty)$, which are evenly distributed on the circle $S(\infty)$ in such a way that we have the following inclusion

$$
\{x \in M: R-1 \leq \rho(x) \leq R+1\} \subset \cup_{i=1}^{N(R)} B_{c_{v_{i}}(R)}\left(3\left(1+\frac{1}{b}\right)\right),
$$

where for each $1 \leq N(R), c_{v_{i}}:[0, \infty) \rightarrow M$ is the geodesic ray of unit speed with $c_{v_{i}}(0)=O$, and $c_{v_{i}}^{\prime}(0)=v_{i}$.

REMARK 4.2. In words, the lemma is saying is that if we consider an annulus in $M$ with inner radius $R-1$ and outer radius $R+1$, where $R$ is big enough, then we can cover it by $N(R)$ geodesic balls centered at $c_{v_{i}}(R)$ and with radius $\left(3\left(1+\frac{1}{b}\right)\right)$.

Proof. To begin, let us select a base point $O$ in $M$, and let $\rho$ be the distance function from $O$. By Lemma 2.3 and Lemma 2.4 there exists a sufficiently large universal constant $R_{0}>1$ such that for any two points $x_{1}, x_{2}$ in $M$ with $R=$ $\left|\overline{O x_{1}}\right|=\left|\overline{O x_{2}}\right|$ satisfying $R \geq R_{0}$, we have the following

$$
2 R+\frac{2}{a}(\log \theta-1) \leq\left|\overline{x_{1} x_{2}}\right| \leq 2 R+\frac{2}{b}(\log \theta+1),
$$

where $\theta=\angle\left(\overline{O x_{1}}, \overline{O x_{2}}\right)$.

From now on, we use the universal constant $\bar{R}_{0}=R_{0}+1$. Now, choose any $R \geq \bar{R}_{0}$, and let $v \in S(\infty)$. We then consider the geodesic ray

$$
\begin{aligned}
& c_{v}:[0, \infty) \rightarrow M, \\
& c_{v}(0)=O, \quad \text { and } \quad c_{v}^{\prime}(0)=v .
\end{aligned}
$$

Now, we consider the universal angle $\theta^{(R)}=e^{-b R}$, and the sector $T_{O}\left(v, \theta^{(R)} ; R-\right.$ $1, R+1)$ defined by

$$
T_{O}\left(v, \theta^{(R)} ; R-1, R+1\right)=\left\{x \in C_{O}\left(v, \theta^{(R)}\right): R-1 \leq \rho(x) \leq R+1\right\},
$$

where the cone $C_{O}\left(v, \theta^{(R))}\right.$ was defined in Section [2.4. Our goal is to prove that $T_{O}\left(v, \theta^{(R)} ; R-1, R+1\right) \subset B_{c_{v}(R)}\left(3\left(1+\frac{1}{b}\right)\right)$. To this end, let $x \in T_{O}\left(v, \theta^{(R)} ; R-\right.$ $1, R+1)$. Then, $\rho(x)=R+\lambda$, for some $\lambda \in[-1,1]$. By the triangle inequality, we have

$$
\left|\overline{c_{v}(R) x}\right| \leq\left|\overline{c_{v}(R) c_{v}(R+\lambda)}\right|+\left|\overline{c_{v}(R+\lambda) x}\right| \leq|\lambda|+\left|\overline{c_{v}(R+\lambda) x}\right| .
$$

But from (4.2) with $x_{1}=c_{v}(R+\lambda)$ and $x_{2}=x$, it follows

$$
\begin{aligned}
\left|\overline{c_{v}(R+\lambda) x}\right| & \leq 2(R+|\lambda|)+\frac{2}{b}\left\{\log \left[\angle\left(\overline{O c_{v}(R+\lambda)}, \overline{O x}\right)\right]+1\right\} \\
& \leq 2 R+2+\frac{2}{b}\left[\log \left(\theta^{(R)}\right)+1\right] \\
& =2 R+2+\frac{2}{b}\left\{\log \left(e^{-b R}\right)+1\right\} \\
& =2 R+2+\frac{2}{b}(-b R+1) \\
& =2+\frac{2}{b}
\end{aligned}
$$

Hence

$$
\left|\overline{c_{v}(R) x}\right| \leq|\lambda|+\left|\overline{c_{v}(R+\lambda) x}\right| \leq|\lambda|+2\left(1+\frac{1}{b}\right)<3\left(1+\frac{1}{b}\right) .
$$


This shows that every $x \in T_{O}\left(v, \theta^{(R)} ; R-1, R+1\right)$ must lie in the geodesic ball $B_{c_{v}(R)}\left(3\left(1+\frac{1}{b}\right)\right)$. To conclude the proof, we just take the integer $N(R)=\left[\frac{2 \pi}{2 e^{-b R}}\right]+1$. Then, we can select some evenly distributed vectors $v_{1}, v_{2}, \ldots, v_{N(R)} \in S(\infty)$ such that

$$
\{x \in M: R-1 \leq \rho(x) \leq R+1\}=\cup_{i=1}^{N(R)} T_{O}\left(v_{i}, \theta^{(R)} ; R-1, R+1\right) .
$$

Since we already know that, for each $1 \leq i \leq N(R)$, we have $T_{O}\left(v_{i}, \theta^{(R)} ; R-1, R+\right.$ 1) $\subset B_{c_{v_{i}}(R)}\left(3\left(1+\frac{1}{b}\right)\right)$, in which $c_{v_{i}}:[0, \infty) \rightarrow M$ is the geodesic with $c_{v_{i}}(0)=O$ and $c_{v_{i}}^{\prime}(0)=v_{i}$, it follows at once from relation (4.6) that

$$
\begin{aligned}
\{x \in M: R-1 \leq \rho(x) \leq R+1\} & =\cup_{i=1}^{N(R)} T_{O}\left(v_{i}, \theta^{(R)} ; R-1, R+1\right) \\
& \subset \cup_{i=1}^{N(R)} B_{c_{v_{i}}(R)}\left(3\left(1+\frac{1}{b}\right)\right),
\end{aligned}
$$

as desired.

With the help of the Covering Lemma 4.1 and Lemma 2.5, we can now prove the following fact.

Proposition 4.3. Let $a, b>0$ satisfy $\frac{1}{2} b<a \leq b$, and let $M$ be a simply connected, complete 2-dimensional Riemannian manifold with sectional curvature $-b^{2} \leq K_{M} \leq-a^{2}$. Then, for any bounded harmonic function $F \in C^{\infty}(M) \cap$ $C^{0}(\bar{M})$, which arises from $C^{1}$ - boundary data $\phi$, it follows that $\left.\int_{M}|\nabla| \nabla F\right|^{2} \mid<\infty$.

REMARK 4.4. We note that in the proof of Proposition 4.3 it is not necessary for us to obtain a uniform bound of $\left.\int_{M}|\nabla| \nabla F\right|^{2} \mid<\infty$ in terms of, say, $\left\|\phi^{\prime}\right\|_{\infty}$. All we need is just to confirm that the integral $\left.\int_{M}|\nabla| \nabla F\right|^{2} \mid$ is finite, because this is already enough to ensure that $\int_{M} \operatorname{div}\left\{\nabla|\nabla F|^{2}\right\}=0$.

Proof. As usual, we begin with a bounded harmonic function $F \in C^{\infty}(M) \cap$ $C^{0}(\bar{M})$ such that $\left.F\right|_{S(\infty)}=\phi$. Let $\bar{R}_{0}$ be the sufficiently large universal constant as determined in Lemma 4.1

Since $F$ is smooth on $M$, in order to prove that $\left.\int_{M}|\nabla| \nabla F\right|^{2} \mid<\infty$, it is sufficient to see that $\left.\int_{M-B_{O}(R)}|\nabla| \nabla F\right|^{2} \mid<\infty$, for some large $R>\bar{R}_{0}$, where $O$ is a selected base point in $M$.

Using (2.14) we first write

$$
\begin{aligned}
\left.|\nabla| \nabla F\right|^{2} \mid & \leq\left|\nabla\left\{|\nabla F|^{2}+A e^{-2 \delta \rho}\right\}\right|+A\left|\nabla e^{-2 \delta \rho}\right| \\
& =\left|\nabla\left\{|\nabla F|^{2}+A e^{-2 \delta \rho}\right\}\right|+A(2 \delta) e^{-2 \delta \rho} .
\end{aligned}
$$

We estimate the first term on the right. First, by (2.22)

$$
\Delta\left[|\nabla F|^{2}\right](x)=2\left[\partial_{i} \partial_{j} F\right]^{2}(x)+2 \partial_{i} F(x) \partial_{i}(\Delta F)(x)+2 \operatorname{Ric}(\nabla F, \nabla F)(x) .
$$

Since $\Delta F=0$ it follows from the above formula that

$$
\Delta\left[|\nabla F|^{2}\right] \geq 2 \operatorname{Ric}(\nabla F, \nabla F) \geq-2 b^{2}|\nabla F|^{2} .
$$

To proceed further, we choose $\delta$, once and for all, to be any fixed positive number within the range $\frac{b}{2}<\delta<a$. Then by Proposition 3.1] we have 


$$
|\nabla F| \leq C e^{-\delta \rho} .
$$

Notice that our fixed choice of $\delta \in\left(\frac{b}{2}, a\right)$ automatically satisfies the condition $\delta>\frac{a}{2}$, due to the fact that $b \geq a$. Next, since $\delta>\frac{b}{2}$, we can choose some sufficiently large positive number depending on $\delta, R(\delta)>2$, such that

$$
\frac{b}{2}<\frac{b}{2}\left(1+\frac{1}{R(\delta)}\right)<\delta<a \leq b .
$$

Next, we have to find some $A>0$ large enough, and some sufficiently large radius $R$ such that the function $|\nabla F|^{2}+A e^{-2 \delta \rho}$ will be subharmonic on $\mathbb{H}^{2}-B_{O}(R)$. To achieve this we use (2.17) and observe

$$
\Delta \rho \chi_{\left\{\rho \geq \frac{R(\delta)}{b}\right\}} \leq b \cdot \operatorname{coth}(b \rho) \chi_{\left\{\rho \geq \frac{R(\delta)}{b}\right\}} \leq\left(b+\frac{1}{\rho}\right) \chi_{\left\{\rho \geq \frac{R(\delta)}{b}\right\}} \leq b\left(1+\frac{1}{R(\delta)}\right) \chi_{\left\{\rho \geq \frac{R(\delta)}{b}\right\}} .
$$

Hence from (4.9), (4.10) and (2.36)

$$
\begin{aligned}
\Delta\left\{|\nabla F|^{2}+A e^{-2 \delta \rho}\right\} \chi_{\left\{\rho \geq \frac{R(\delta)}{b}\right\}} & \geq\left\{-2 b^{2} C^{2} e^{-2 \delta \rho}+A(2 \delta) e^{-2 \delta \rho}[2 \delta-\Delta \rho]\right\} \chi_{\left\{\rho \geq \frac{R(\delta)}{b}\right\}} \\
& \geq 2\left\{A \delta\left[2 \delta-b\left(1+\frac{1}{R(\delta)}\right)\right]-b^{2} C^{2}\right\} e^{-2 \delta \rho} \chi_{\left\{\rho \geq \frac{R(\delta)}{b}\right\}} .
\end{aligned}
$$

Notice $2 \delta-b\left(1+\frac{1}{R(\delta)}\right)>0$, thanks to our choice of $R(\delta)$ which ensures the survival of the second inequality sign in (4.11). Next, we just take

$$
A=\frac{2 b^{2} C^{2}}{\delta\left[2 \delta-b\left(1+\frac{1}{R(\delta)}\right)\right]} .
$$

With this choice of $A$, it follows from (4.13) that

$$
\Delta\left\{|\nabla F|^{2}+A e^{-2 \delta \rho}\right\} \chi_{\left\{\rho \geq \frac{R(\delta)}{b}\right\}} \geq 0 .
$$

That is, the function $|\nabla F|^{2}+A e^{-2 \delta \rho}$ is subharmonic on $M-B_{O}\left(\frac{R(\delta)}{b}\right)$. So, we may apply Lemma 2.5 to $|\nabla F|^{2}+A e^{-2 \delta \rho}$ and deduce that for any geodesic ball $B_{x}\left(6\left(1+\frac{1}{b}\right)\right) \subset M-B_{O}\left(\frac{R(\delta)}{b}\right)$, we have

$$
\begin{aligned}
& \int_{B_{x}\left(3\left(1+\frac{1}{b}\right)\right)}\left|\nabla\left\{|\nabla F|^{2}+A e^{-2 \delta \rho}\right\}\right| \\
& \leq\left|B_{x}\left(3\left(1+\frac{1}{b}\right)\right)\right|^{\frac{1}{2}}\left\{\int_{B_{x}\left(3\left(1+\frac{1}{b}\right)\right)}\left|\nabla\left\{|\nabla F|^{2}+A e^{-2 \delta \rho}\right\}\right|^{2}\right\}^{\frac{1}{2}} \\
& \leq C_{b}\left\{\int_{B_{x}\left(6\left(1+\frac{1}{b}\right)\right)}\left[|\nabla F|^{2}+A e^{-2 \delta \rho}\right]^{2}\right\}^{\frac{1}{2}} \\
& \leq C e^{-2 \delta \rho(x)},
\end{aligned}
$$

where we again used (4.10) to go to the last line. We also employed the volume comparison theorem, which says that $K_{M} \geq-b^{2}$ implies that the volume of any 
geodesic ball $B_{x}\left(6\left(1+\frac{1}{b}\right)\right)$ in $M$ is bounded above by a universal constant $C_{b}$. Indeed, $C_{b}$ is the constant volume of any geodesic ball with radius $6\left(1+\frac{1}{b}\right)$ in the space form $\mathbb{H}^{2}\left(-b^{2}\right)$.

Now, let us take $K_{0}$ to be a sufficiently large positive integer for which $K_{0} \geq$ $\max \left\{\bar{R}_{0}, \frac{R(\delta)}{b}+6\left(1+\frac{1}{b}\right)\right\}$, where $\bar{R}_{0}$ is the sufficiently large universal constant determined in Lemma 4.1 Then, by Lemma 4.1 for any positive integer $k \geq K_{0}$, if we take the positive integer $N(k)=\left[\pi e^{b k}\right]+1$ then, we can pick a list of vectors $v_{k, 1}, v_{k, 2}, v_{k, 3}, \ldots . v_{k, N(k)} \in S(\infty)$ in such a way that we have the following inclusion

$$
\{x \in M: k-1 \leq \rho(x) \leq k+1\} \subset \cup_{i=1}^{N(k)} B_{c_{v_{k, i}}(k)}\left(3\left(1+\frac{1}{b}\right)\right) .
$$

By combining inequality (4.16) and the above inclusion, it follows that

$$
\begin{aligned}
\int_{\left\{\rho(x) \geq K_{0}\right\}}\left|\nabla\left\{|\nabla F|^{2}+A e^{-2 \delta \rho}\right\}\right| & \leq \sum_{k=K_{0}}^{\infty} \int_{\{k-1 \leq \rho(x) \leq k+1\}}\left|\nabla\left\{|\nabla F|^{2}+A e^{-2 \delta \rho}\right\}\right| \\
& \leq \sum_{k=K_{0}}^{\infty} \sum_{i=1}^{N(k)} \int_{B_{c_{v_{k, i}}(k)}\left(3\left(1+\frac{1}{b}\right)\right)}\left|\nabla\left\{|\nabla F|^{2}+A e^{-2 \delta \rho}\right\}\right| \\
& \leq \sum_{k=K_{0}}^{\infty} \sum_{i=1}^{N(k)} C e^{-2 \delta k} \\
& \leq \sum_{k=K_{0}}^{\infty} C\left\{\left[\pi e^{b k}\right]+1\right\} e^{-2 \delta k} \\
& <\infty .
\end{aligned}
$$

We note that to obtain the last inequality $\sum_{k=K_{0}}^{\infty} C\left\{\left[\pi e^{b k}\right]+1\right\} e^{-2 \delta k}<\infty$, we use the fact that our fixed choice of $\delta$ lies within the range $\frac{1}{2} b<\delta<a$, so that $(2 \delta-b)>0$ is automatic, and hence $\sum_{k=K_{0}}^{\infty} e^{b k} e^{-2 \delta k}=\sum_{k=K_{0}}^{\infty} e^{-(2 \delta-b) k}<\infty$.

Next, we notice that the volume form on $M$ (with respect to the geodesic normal polar coordinates $(r, \theta)$ about $O)$ is in the form of $G(r, \theta) d r d \theta$, where $G(r, \theta) \leq$ $\frac{1}{b} \sinh (b r)$ thanks to the comparison theorem 2.8 for Jacobi fields (with $K_{M} \geq-b^{2}$ ). Hence, it follows again from $2 \delta>b$ that

$$
\begin{aligned}
\int_{M} e^{-2 \delta \rho} & =\int_{0}^{2 \pi} \int_{0}^{\infty} e^{-2 \delta r} G(r, \theta) d r d \theta \\
& \leq \int_{0}^{2 \pi} \int_{0}^{\infty} e^{-2 \delta r} \frac{1}{b} \sinh (b r) d r d \theta<\infty .
\end{aligned}
$$

Hence by (4.8), (4.17) and (4.18)

$$
\begin{aligned}
& \left.\int_{\left\{\rho(x) \geq K_{0}\right\}}|\nabla| \nabla F\right|^{2}\left|\leq \int_{\left\{\rho(x) \geq K_{0}\right\}}\right| \nabla\left\{|\nabla F|^{2}+A e^{-2 \delta \rho}\right\} \mid \\
& +A(2 \delta) \int_{\left\{\rho(x) \geq K_{0}\right\}} e^{-2 \delta \rho}<\infty .
\end{aligned}
$$

Since $\left.|\nabla| \nabla F\right|^{2} \mid$ is continuous in $M$, by (4.19) we must have $\left.\int_{M}|\nabla| \nabla F\right|^{2} \mid<\infty$ as needed. 


\section{Finite Dissipation}

We begin with two propositions, which help us establish the energy inequality (1.4). First, using $\bar{g}(\cdot, \cdot)$ on $T^{*} M \otimes T^{*} M$ defined in Section 2 we can consider for each 1-form $\theta$, two non-negative valued functions $\bar{g}(\operatorname{Def} \theta$, $\operatorname{Def} \theta) \in C^{\infty}(M)$, and $\bar{g}(\bar{\nabla} \theta, \bar{\nabla} \theta) \in C^{\infty}(M)$. We have the following relationship between them.

Lemma 5.1. For any given n-dimensional complete Riemannian manifold $M$, we have

$$
\bar{g}(\operatorname{Def} \theta, \operatorname{Def} \theta) \leq \bar{g}(\bar{\nabla} \theta, \bar{\nabla} \theta) .
$$

Proof. Let $p \in M$, and consider the geodesic normal coordinates $\left(x^{1}, x^{2}, \ldots, x^{n}\right)$ about the point $p$, so that the natural frame $\left.\partial_{1}\right|_{p},\left.\partial_{2}\right|_{p}, \ldots,\left.\partial_{n}\right|_{p}$ (induced by the geodesic normal coordinates) at the point $p$ is orthonormal, and that the Christoffel symbols $\Gamma_{j k}^{l}$ (induced by the geodesic normal coordinate) vanish at the point $p$. Hence, for any 1 -form $\theta=\sum_{j} \theta_{j} d x^{j}$ we have $\theta_{j ; k}(p)=\partial_{k} \theta_{j}(p)$. So, by [2.9] it follows

$$
\begin{aligned}
& \left.\bar{g}(\operatorname{Def} \theta, \operatorname{Def} \theta)\right|_{p} \\
& =\frac{1}{4} \bar{g}\left(\left.\sum_{i, j}\left(\partial_{i} \theta_{j}+\partial_{j} \theta_{i}\right)(p) d x^{i} \otimes d x^{j}\right|_{p},\left.\sum_{k, l}\left(\partial_{k} \theta_{l}+\partial_{l} \theta_{k}\right)(p) d x^{k} \otimes d x^{l}\right|_{p}\right) \\
& =\frac{1}{4} \sum_{i, j} \sum_{k, l}\left(\partial_{i} \theta_{j}+\partial_{j} \theta_{i}\right)(p)\left(\partial_{k} \theta_{l}+\partial_{l} \theta_{k}\right)(p) \delta^{i k} \delta^{j l} \\
& =\frac{1}{4} \sum_{i, j}\left(\partial_{i} \theta_{j}+\partial_{j} \theta_{i}\right)(p)\left(\partial_{i} \theta_{j}+\partial_{j} \theta_{i}\right)(p) \\
& \leq \frac{1}{2} \sum_{i, j}\left(\partial_{i} \theta_{j}(p)\right)^{2}+\left(\partial_{j} \theta_{i}(p)\right)^{2} \\
& =\sum_{i, j}\left(\partial_{i} \theta_{j}(p)\right)^{2} .
\end{aligned}
$$

On the other hand, by (2.13) the Christoffel symbols $\Gamma_{j k}^{l}$ vanish at $p$, so it follows that $\left.\bar{\theta}\right|_{p}=\left.\sum_{i, j} \partial_{i} \theta_{j}(p) d x^{i} \otimes d x^{j}\right|_{p}$. Hence

$$
\left.\bar{g}(\bar{\nabla} \theta, \bar{\nabla} \theta)\right|_{p}=\sum_{i, j}\left(\partial_{i} \theta_{j}(p)\right)^{2},
$$

and

$$
\left.\bar{g}(\operatorname{Def} \theta, \operatorname{Def} \theta)\right|_{p} \leq \sum_{i, j}\left(\partial_{i} \theta_{j}(p)\right)^{2}=\left.\bar{g}(\bar{\nabla} \theta, \bar{\nabla} \theta)\right|_{p} .
$$

Since $p \in M$ is arbitrary in the above argument, it follows that the above inequality is valid for all points in $M$ as needed.

COROLlary 5.2. For any given n-dimensional complete Riemannian manifold $M$, if $\theta=d F$, where $F$ is any $C^{2}(M)$ function, we have

$$
\bar{g}(\operatorname{Def} \theta, \operatorname{Def} \theta)=\bar{g}(\bar{\nabla} \theta, \bar{\nabla} \theta) .
$$

Proof. The inspection of the above proof shows that in the only place where we had inequality we could actually just do a direct computation to obtain equality with the next line, and not use Cauchy's inequality. 
Proposition 5.3. Let $a, b>0$ such that $\frac{1}{2} b<a \leq b$. Let $M$ be a simply connected, complete 2-dimensional Riemannian manifold with sectional curvature $-b^{2} \leq K_{M} \leq-a^{2}$. Let $\phi \in C^{1}(S(\infty))$ be any given boundary data, and let $F \in$ $C^{\infty}(M) \cap C^{0}(\bar{M})$ be the unique bounded harmonic function on $\bar{M}$ with $\left.F\right|_{S(\infty)}=\phi$. Then the following holds

$$
\int_{M} \bar{g}(\bar{\nabla}(d F), \bar{\nabla}(d F))=-\int_{M} \operatorname{Ric}(\nabla F, \nabla F) \leq b^{2} \int_{M}|\nabla F|^{2} .
$$

Proof. Let $F \in C^{\infty}(M) \cap C^{0}(\bar{M})$ be the unique bounded harmonic function on $M$ with $\left.F\right|_{S(\infty)}=\phi$, where $\phi \in C^{1}(S(\infty))$ is some given boundary data.

Then again by 2.22)

$\Delta\left(|\nabla F|^{2}\right)(x)=2 \sum_{1 \leq i, j \leq 2}\left[\partial_{i} \partial_{j} F\right]^{2}(x)+2 \sum_{1 \leq i, j \leq 2} \partial_{i} F(x) \partial_{i}(\Delta F)(x)+2 \operatorname{Ric}(\nabla F, \nabla F)(x)$,

where $\partial_{1}, \partial_{2}$ is the natural coordinate frame induced by the geodesic normal coordinates about the point $x$. So again by (2.13) Christoffel symbols $\Gamma_{j k}^{l}$ vanish at $x$, and we have

$$
\sum_{1 \leq i, j \leq 2}\left[\partial_{i} \partial_{j} F\right]^{2}(x)=\left.\bar{g}(\bar{\nabla}(d F), \bar{\nabla}(d F))\right|_{x} .
$$

Also, as before we use $\Delta F=0$ in (5.4) to obtain

$$
\frac{1}{2} \Delta\left(|\nabla F|^{2}\right)(x)=\bar{g}(\bar{\nabla}(d F), \bar{\nabla}(d F))(x)+\operatorname{Ric}(\nabla F, \nabla F)(x) \quad \forall x \in M .
$$

Now, for each positive integer $k \geq 1$, consider a smooth function $\psi_{k}:[0, \infty) \rightarrow$ $\mathbb{R}$, which satisfies $\chi_{\left[0,2^{k}\right]} \leq \psi_{k} \leq \chi_{\left[0,2^{k+1}\right]}$, and $\left|\psi_{k}^{\prime}\right| \leq \frac{2}{2^{k}}$. Now, let $O$ be a selected base point in $M$, and let $\rho$ be the distance function from $O$. Then, by multiplying the above equality by the cut off function $\psi_{k}(\rho)$ and integrating over $M$, we yield the following equality

$$
\int_{M} \frac{1}{2} \operatorname{div}\left(\nabla|\nabla F|^{2}\right) \psi_{k}(\rho)=\int_{M} \psi_{k}(\rho) \bar{g}(\bar{\nabla}(d F), \bar{\nabla}(d F))+\int_{M} \psi_{k}(\rho) \operatorname{Ric}(\nabla F, \nabla F) .
$$

But since $\psi_{k}(\rho)$ is compactly supported in $B_{O}\left(2^{k+1}\right)$, it is plain to see that, for every $k \geq 1$, we have

$$
\begin{aligned}
\left|\int_{M} \frac{1}{2} \operatorname{div}\left(\nabla|\nabla F|^{2}\right) \psi_{k}(\rho)\right| & =\frac{1}{2}\left|\int_{M} 2 \psi_{k}^{\prime}(\rho) \nabla \rho \cdot \nabla\left(|\nabla F|^{2}\right)\right| \\
& \leq \frac{2}{2^{k}} \int_{M}\left|\nabla\left(|\nabla F|^{2}\right)\right| .
\end{aligned}
$$

Since, according to Proposition 4.3 we have $\int_{M}\left|\nabla\left(|\nabla F|^{2}\right)\right|<\infty$, it follows from the above inequality that

$$
\lim _{k \rightarrow \infty} \int_{M} \frac{1}{2} \operatorname{div}\left(\nabla|\nabla F|^{2}\right) \psi_{k}(\rho)=0 .
$$

On the other hand, by the monotone convergence theorem, we have

$$
\lim _{k \rightarrow \infty} \int_{M} \psi_{k}(\rho) \bar{g}(\bar{\nabla}(d F), \bar{\nabla}(d F))=\int_{M} \bar{g}(\bar{\nabla}(d F), \bar{\nabla}(d F)),
$$

and that 


$$
\lim _{k \rightarrow \infty} \int_{M} \psi_{k}(\rho) \cdot(-\operatorname{Ric}(\nabla F, \nabla F))=\int_{M}(-\operatorname{Ric}(\nabla F, \nabla F))
$$

As a result, by taking the limit of each side in equality (5.5), we get

$$
0=\int_{M} \bar{g}(\bar{\nabla}(d F), \bar{\nabla}(d F))+\int_{M} \operatorname{Ric}(\nabla F, \nabla F) .
$$

That is, we have

$$
\int_{M} \bar{g}(\bar{\nabla}(d F), \bar{\nabla}(d F))=-\int_{M} \operatorname{Ric}(\nabla F, \nabla F) \leq b^{2} \int_{M}|\nabla F|^{2},
$$

in which the last inequality follows from the fact (2.11) that $\operatorname{Ric}(\nabla F, \nabla F)=$ $K_{M}|\nabla F|^{2} \geq-b^{2}|\nabla F|^{2}$.

Corollary 5.4. Let $a>0$ and let $\phi \in C^{1}(S(\infty))$ be any given boundary data, and let $F \in C^{\infty}\left(\mathbb{H}^{2}\left(-a^{2}\right)\right) \cap C^{0}\left(\overline{\mathbb{H}^{2}\left(-a^{2}\right)}\right)$ be the unique bounded harmonic function on $\overline{\mathbb{H}^{2}\left(-a^{2}\right)}$ with $\left.F\right|_{S(\infty)}=\phi$. Then the following holds

$$
\int_{\mathbb{H}^{2}\left(-a^{2}\right)} \bar{g}(\bar{\nabla}(d F), \bar{\nabla}(d F))=a^{2} \int_{\mathbb{H}^{2}\left(-a^{2}\right)}|\nabla F|^{2} .
$$

Proof. This is immediate from equation (5.3) since $\operatorname{Ric}(\nabla F, \nabla F)=-a^{2}|\nabla F|^{2}$.

\section{Proofs of the main results}

First we establish the following lemma. A simpler computation in normal coordinates could also be done in the same spirit as the computation in the Euclidean space. However, we present a different proof below due to its intrinsic nature.

LEMma 6.1. The following identity is valid for any smooth function $f$ on any given $n$-dimensional Riemannian manifold $M$.

$$
\bar{\nabla}_{\nabla f} d f=\frac{1}{2} d|d f|^{2} .
$$

Proof. First, for any smooth vector field $X$ on a Riemannian manifold $M$, and for any smooth function $f$ on $M$, we write

$$
X(f)=\langle X, d f\rangle_{T M \otimes T^{*} M} .
$$

Next, recall that the Lie bracket $[X, Y]$ between two vector fields $X$ and $Y$, is itself another vector field, and is characterized by $[X, Y](f)=X(Y(f))-Y(X(f))$, for any $f \in C^{\infty}(M)$.

Now, for a given smooth function $f$ on a Riemannian manifold $M$, we consider the gradient field $W=\nabla f$, which means that $W^{*}=d f$. Then, by (2.1) it is plain to see that

$$
W(f)=\langle W, d f\rangle_{T M \otimes T^{*} M}=g(W, W)=|W|^{2} .
$$

Next, we have the following identity for any smooth vector field $X$ on $M$, due to the fact that the Levi-Civita connection $\bar{\nabla}$ on $T M$ is compatible with the Riemannian metric $g(\cdot, \cdot)$ on $M$ we have

$$
\frac{1}{2} X\left(|W|^{2}\right)=\frac{1}{2}\left\langle X, d\left(|W|^{2}\right)\right\rangle_{T M \otimes T^{*} M}=g\left(\bar{\nabla}_{X} W, W\right),
$$


and

$$
\begin{aligned}
\left\langle X, \bar{\nabla}_{W} W^{*}\right\rangle_{T M \otimes T^{*} M} & =\left\langle X,\left(\bar{\nabla}_{W} W\right)^{*}\right\rangle_{T M \otimes T^{*} M} \quad \text { by 2.6) } \\
& =g\left(X, \bar{\nabla}_{W} W\right) \quad \text { by (2.1) } \\
& =W(g(X, W))-g\left(\bar{\nabla}_{W} X, W\right) \quad \text { by compatibility } \\
& =W\left(\langle X, d f\rangle_{T M \otimes T^{*} M}\right)-g\left(\bar{\nabla}_{W} X, W\right) \\
& =W(X(f))-g\left(\bar{\nabla}_{W} X, W\right) .
\end{aligned}
$$

But due to the torsion free property of the Levi-Civita connection $\bar{\nabla}$ on $T M$, which says that $-\bar{\nabla}_{W} X=[X, W]-\bar{\nabla}_{X} W$, we have

$$
-g\left(\bar{\nabla}_{W} X, W\right)=g([X, W], W)-g\left(\bar{\nabla}_{X} W, W\right) .
$$

Hence by (6.4)

$$
\begin{aligned}
\left\langle X, \bar{\nabla}_{W} W^{*}\right\rangle_{T M \otimes T^{*} M} & =W(X(f))+g([X, W], W)-g\left(\bar{\nabla}_{X} W, W\right) \\
& =W(X(f))+[X, W](f)-\frac{1}{2} X\left(|W|^{2}\right) \quad \text { by (2.1) and [6.3) } \\
& =W(X(f))+X(W(f))-W(X(f))-\frac{1}{2} X\left(|W|^{2}\right) \\
& =\frac{1}{2} X\left(|W|^{2}\right),
\end{aligned}
$$

where the last equality follows since $X(W(f))=X\left(|W|^{2}\right)$ by 6.2 . In conclusion, by using (6.3) again, the following equality holds for any smooth vector field $X$ on $M$

$$
<X, \bar{\nabla}_{W} W^{*}-\frac{1}{2} d\left(|W|^{2}\right)>_{T M \otimes T^{*} M}=0,
$$

which means the same as saying that $\bar{\nabla}_{\nabla f} d f-\frac{1}{2} d\left(|d f|^{2}\right)=0$ as needed.

6.1. Proof of Theorem 1.3. First we show existence and the lack of uniqueness.

Existence and Non-uniqueness: For convenience we recall the Navier-Stokes equation on $\mathbb{H}^{2}\left(-a^{2}\right)$.

$$
\begin{aligned}
\partial_{t} U^{*}-\Delta U^{*}+\bar{\nabla}_{U} U^{*}-2 \operatorname{Ric}\left(U^{*}\right)+d P & =0 . \\
d^{*} U^{*} & =0
\end{aligned}
$$

Now, let $\phi \in C^{0}(S(\infty))$, then by Theorem 2.9 there exists a (unique) harmonic function $F \in C^{\infty}\left(\mathbb{H}^{2}\left(-a^{2}\right)\right) \cap C^{0}\left(\overline{\mathbb{H}^{2}\left(-a^{2}\right)}\right)$ satisfying $\left.F\right|_{S(\infty)}=\phi$. We let our initial data $u_{0}=d F$, and define a solution $\left(U^{*}, P\right)$ to be

$$
U^{*}=\psi(t) d F, \quad P=-\partial_{t} \psi(t) F-\frac{1}{2} \psi^{2}(t)|d F|^{2}-2 a^{2} \psi(t) F
$$

where $\psi$ is any function satisfying

$$
\psi^{2}(t)+4 a^{2} \int_{0}^{t} \psi^{2}(s) d s \leq \psi^{2}(0) .
$$

For example, we could let $\psi(t)=\exp \left(-\frac{A t}{2}\right)$ for any $A \geq 4 a^{2}$.

First we show $\left(U^{*}, P\right)$ solves $\mathrm{N}_{-} \mathrm{S}_{\mathbb{H}^{2}\left(-a^{2}\right)}$. This is very easy by the preparations 
we have done in Section 2.6 Indeed, by (2.42) $\Delta U^{*}=0$, and we observe that by Lemma 6.1] and (2.12),

$$
\partial_{t} U^{*}+\bar{\nabla}_{U} U^{*}-2 \operatorname{Ric}\left(U^{*}\right)=-d P .
$$

It is also very easy to see that $U^{*}$ is divergence free since by definition of $U^{*}$ and (2.41) this is equivalent to $F$ being harmonic.

Global energy inequality: Recall we want to show

$$
\int_{\mathbb{H}^{2}\left(-a^{2}\right)}\left|U^{*}(t, x)\right|^{2}+4 \int_{0}^{t} \int_{\mathbb{H}^{2}\left(-a^{2}\right)} \bar{g}\left(\operatorname{Def} U^{*}, \operatorname{Def} U^{*}\right)(s, x) d s \leq \int_{\mathbb{H}^{2}\left(-a^{2}\right)}\left|u_{0}\right|^{2} .
$$

Thanks to Corollary 5.2 we have

$$
\begin{aligned}
\int_{\mathbb{H}^{2}\left(-a^{2}\right)}\left|U^{*}(t, x)\right|^{2}+4 \int_{0}^{t} \int_{\mathbb{H}^{2}\left(-a^{2}\right)} \bar{g}\left(\operatorname{Def} U^{*}, \operatorname{Def} U^{*}\right)(s, x) d s \\
=\int_{\mathbb{H}^{2}\left(-a^{2}\right)}\left|U^{*}(t, x)\right|^{2}+4 \int_{0}^{t} \int_{\mathbb{H}^{2}\left(-a^{2}\right)} \bar{g}\left(\bar{\nabla} U^{*}, \bar{\nabla} U^{*}\right)(s, x) d s .
\end{aligned}
$$

By Corollary 5.4

$$
\int_{\mathbb{H}^{2}\left(-a^{2}\right)} \bar{g}(\bar{\nabla}(d F), \bar{\nabla}(d F))=a^{2} \int_{\mathbb{H}^{2}\left(-a^{2}\right)}|\nabla F(t, x)|^{2},
$$

so

$$
\int_{\mathbb{H}^{2}\left(-a^{2}\right)} \bar{g}\left(\bar{\nabla} U^{*}, \bar{\nabla} U^{*}\right)=a^{2} \int_{\mathbb{H}^{2}\left(-a^{2}\right)} \psi^{2}(t)|\nabla F(t, x)|^{2} .
$$

Hence by 2.3 and (6.6)

$$
\begin{aligned}
\int_{\mathbb{H}^{2}\left(-a^{2}\right)}\left|U^{*}(t, x)\right|^{2}+4 \int_{0}^{t} \int_{\mathbb{H}^{2}\left(-a^{2}\right)} \bar{g}\left(\bar{\nabla} U^{*}, \bar{\nabla} U^{*}\right) d s \\
\quad=\int_{\mathbb{H}^{2}\left(-a^{2}\right)} \psi^{2}(t)|\nabla F(x)|^{2}+4 a^{2} \int_{0}^{t} \int_{\mathbb{H}^{2}\left(-a^{2}\right)} \psi^{2}(s)|\nabla F(x)|^{2} d s \\
=\left(\psi^{2}(t)+4 a^{2} \int_{0}^{t} \psi^{2}(s) d s\right) \int_{\mathbb{H}^{2}\left(-a^{2}\right)}|\nabla F(x)|^{2} \\
\leq \psi^{2}(0) \int_{\mathbb{H}^{2}\left(-a^{2}\right)}|\nabla F(x)|^{2} \\
\quad=\int_{\mathbb{H}^{2}\left(-a^{2}\right)}\left|u_{0}\right|^{2},
\end{aligned}
$$

as needed.

REMARK 6.2. We make a brief remark that in fact, the energy inequality could be derived directly from the equation as follows. By starting with the equation for $\left(U^{*}, P\right)$ as above and using that $\Delta U^{*}=0$ we have

$$
\partial_{t} U^{*}+\bar{\nabla}_{U} U^{*}-2 \operatorname{Ric} U^{*}+d P=0 .
$$

We further cancel out $\bar{\nabla}_{U} U^{*}$ with a part of the $d P$ leaving

$$
\partial_{t} U^{*}-2 \operatorname{Ric} U^{*}-\partial_{t} U^{*}-2 a^{2} U^{*}=0 .
$$


Let $\langle\cdot, \cdot\rangle$ denote $\bar{g}(\cdot, \cdot)$, then by (6.7)

$$
\begin{aligned}
0 & =\left\langle\partial_{t} U^{*}, U^{*}\right\rangle-\left\langle 2 \operatorname{Ric} U^{*}, U^{*}\right\rangle+\left\langle\partial_{t} U^{*}-2 a^{2} U^{*}, U^{*}\right\rangle \\
& =\frac{1}{2} \partial_{t}\left|U^{*}\right|^{2}+2 a^{2} \psi^{2}\langle d F, d F\rangle+\left(\frac{A}{2}-2 a^{2}\right) \psi^{2}\langle d F, d F\rangle \\
& \geq \frac{1}{2} \partial_{t}\left|U^{*}\right|^{2}+2 a^{2} \psi^{2}\langle d F, d F\rangle,
\end{aligned}
$$

if $A \geq 4 a^{2}$, which is what we assume. We now integrate both in time and space. To obtain the dissipation we use Corollary 5.4 and Lemma [5.1]

6.2. Proof of Theorem 1.7. The proof is very similar. Therefore we just give a brief sketch.

Existence and Non-uniqueness: Again, for convenience, we recall the modified Navier-Stokes equation on $M$.

$$
\begin{aligned}
\partial_{t} U^{*}-\Delta U^{*}+\bar{\nabla}_{U} U^{*}+d P & =0, \\
d^{*} U^{*} & =0 .
\end{aligned}
$$

Let $u_{0}=d F$, and

$$
U^{*}=\psi(t) d F, \quad P=-\partial_{t} \psi(t) F-\frac{1}{2} \psi^{2}(t)|d F|^{2},
$$

with $\psi(t)=\exp \left(-\frac{A t}{2}\right)$ for any $A>4 b^{2}$.. Then as before we can see the equation is satisfied.

Global energy inequality: By Corollary 5.2 we need to establish

$$
\int_{M}\left|U^{*}(t, x)\right|^{2}+4 \int_{0}^{t} \int_{M} \bar{g}\left(\bar{\nabla} U^{*}, \bar{\nabla} U^{*}\right) d s \leq \int_{M}\left|u_{0}\right|^{2} .
$$

By Proposition 5.3

$$
\int_{M} \bar{g}(\bar{\nabla}(d F), \bar{\nabla}(d F)) \leq b^{2} \int_{M}|\nabla F|^{2},
$$

so

$$
\int_{M} \bar{g}\left(\bar{\nabla} U^{*}, \bar{\nabla} U^{*}\right) \leq b^{2} \int_{M} \psi^{2}(t)|\nabla F(t, x)|^{2}
$$

Hence

$$
\begin{aligned}
\int_{M} \mid U^{*}(t & , x)\left.\right|^{2}+4 \int_{0}^{t} \int_{M} \bar{g}\left(\bar{\nabla} U^{*}, \bar{\nabla} U^{*}\right) d s \\
& \leq \int_{M} \psi^{2}(t)|\nabla F(x)|^{2}+4 b^{2} \int_{0}^{t} \int_{M}|\psi(s) \nabla F(x)|^{2} d s \\
& =\left(\psi^{2}(t)+4 b^{2} \int_{0}^{t} \psi^{2}(s) d s\right) \int_{M}|\nabla F(x)|^{2} \\
& \leq \psi^{2}(0) \int_{M}|\nabla F(x)|^{2} \\
& =\int_{M}\left|u_{0}\right|^{2}
\end{aligned}
$$

as needed. 
6.3. Proof of Corollary 1.5 and 1.9. In the recent paper [16, Koch, Nadirashvili, Seregin and Šverák studied Liouville thorems and their consequences for the Navier-Stokes equation. One of the results is

TheOrem. 16 Let u be a bounded weak solution of the Navier-Stokes equation on $\mathbb{R}^{2} \times(-\infty, 0)$. Then $u(x, t)=b(t)$ for a suitable bounded measurable function $b:(\infty, 0) \rightarrow \mathbb{R}^{2}$.

The three dimensional problem is more difficult. Nevertheless Koch, Nadirashvili, Seregin and Šverák are able to obtain corresponding results for the axisymmetric equations with no swirl. What Corollaries 1.5 and 1.9 show that in the hyperbolic setting we can have bounded solutions in both two and three dimensions (in fact, for any $n \geq 2$ ) that are not functions of time only. The nontrivial bounded solutions we choose are in the form of (6.5)

$$
U^{*}=\psi(t) d F, \quad P=-\partial_{t} \psi(t) F-\frac{1}{2} \psi^{2}(t)|d F|^{2}-2 a^{2}(n-1) \psi(t) F,
$$

for $\mathrm{N}_{-} \mathrm{S}_{H^{n}\left(-a^{2}\right)}$, and of the form (6.11) for (1.3). However, now we do not need the special form of $\psi$. All we require of $\psi$ is that it is $C^{1}$ and bounded.

It would be interesting to find out whether or not these are the only bounded solutions of $\mathrm{N}-\mathrm{S}_{H^{n}\left(-a^{2}\right)}$ and (1.3) .

Here we also mention the result of Galdi [6], which states

Theorem. 6 For the steady Navier-Stokes equation on $\mathbb{R}^{3}$ whenever the solution satisfies the finite dissipation property and $u \in L_{x}^{9 / 2}$, then $u$ must be a trivial solution, i.e. $u$ is constant.

We note that in our case, we have a nontrivial solution, which belongs to $L_{x}^{9 / 2}$, but at this time we cannot say whether or not there exist nontrivial solutions in three dimensions that also satisfy the finite dissipation property.

Proof of Corollary 1.5 and 1.9 Let $n \geq 2$. Let $\psi$ be bounded, and $C^{1}$ in (6.12) and in (6.11) and without the condition (6.6). It is obvious from the proof of Theorems 1.3 and 1.7 that (6.12) and (6.11) are the solutions of $\mathrm{N}-\mathrm{S}_{H^{n}}\left(-a^{2}\right)$ and (1.3) respectively since this is independent of the dimension of the underlying manifold. Hence, we only need to verify that $U^{*}$ is $L^{\infty}$ bounded. That can be checked in more than one way as follows, we could use the gradient estimate, Theorem 2.6] and bound $|d F|$ by $F$, which is bounded by definition or we could use the exponential decay of the gradient, estimate (3.1).

\section{References}

[1] Michael T. Anderson. The Dirichlet problem at infinity for manifolds of negative curvature. J. Differential Geom., 18(4):701-721 (1984), 1983.

[2] Michael T. Anderson and Richard Schoen. Positive harmonic functions on complete manifolds of negative curvature. Ann. of Math. (2), 121(3):429-461, 1985.

[3] A. Avez and Y. Bamberger. Mouvements sphériques des fluides visqueux incompressibles. $J$. Mécanique, 17(1):107-145, 1978.

[4] Martin Dindoš and Marius Mitrea. The stationary Navier-Stokes system in nonsmooth manifolds: the Poisson problem in Lipschitz and $C^{1}$ domains. Arch. Ration. Mech. Anal., 174(1):1$47,2004$.

[5] David G. Ebin and Jerrold Marsden. Groups of diffeomorphisms and the motion of an incompressible fluid. Ann. of Math. (2), 92:102-163, 1970. 
[6] Giovanni P. Galdi. An introduction to the mathematical theory of the Navier-Stokes equations. Vol. II, volume 39 of Springer Tracts in Natural Philosophy. Springer-Verlag, New York, 1994. Nonlinear steady problems.

[7] David Gilbarg and Neil S. Trudinger. Elliptic partial differential equations of second order. Classics in Mathematics. Springer-Verlag, Berlin, 2001. Reprint of the 1998 edition.

[8] Eberhard Hopf. Über die Anfangswertaufgabe für die hydrodynamischen Grundgleichungen. Math. Nachr., 4:213-231, 1951.

[9] Loo Keng Hua. Starting with the unit circle. Springer-Verlag, New York, 1981. Background to higher analysis, Translated from the Chinese by Kuniko Weltin.

[10] A. A. Il'in. Navier-Stokes and Euler equations on two-dimensional closed manifolds. Mat. Sb., 181(4):521-539, 1990.

[11] A. A. Il'in and A. N. Filatov. Unique solvability of the Navier-Stokes equations on a twodimensional sphere. Dokl. Akad. Nauk SSSR, 301(1):18-22, 1988.

[12] L. Iskauriaza, G. A. Serëgin, and V. Shverak. $L_{3, \infty}$-solutions of Navier-Stokes equations and backward uniqueness. Uspekhi Mat. Nauk, 58(2(350)):3-44, 2003.

[13] Jürgen Jost. Riemannian geometry and geometric analysis. Universitext. Springer-Verlag, Berlin, fifth edition, 2008.

[14] Dominic D. Joyce. Compact manifolds with special holonomy. Oxford Mathematical Monographs. Oxford University Press, Oxford, 2000.

[15] Boris Khesin and Gerard Misiołek. The Euler and Navier-Stokes equations on the hyperbolic plane. Proc. Nat. Acad. Sci., 2012.

[16] Gabriel Koch, Nikolai Nadirashvili, Gregory A. Seregin, and Vladimir Šverák. Liouville theorems for the Navier-Stokes equations and applications. Acta Math., 203(1):83-105, 2009.

[17] O. A. Ladyženskaja. Uniqueness and smoothness of generalized solutions of Navier-Stokes equations. Zap. Naučn. Sem. Leningrad. Otdel. Mat. Inst. Steklov. (LOMI), 5:169-185, 1967.

[18] O. A. Ladyzhenskaya. The mathematical theory of viscous incompressible flow. Second English edition, revised and enlarged. Translated from the Russian by Richard A. Silverman and John Chu. Mathematics and its Applications, Vol. 2. Gordon and Breach Science Publishers, New York, 1969.

[19] John M. Lee. Riemannian manifolds, volume 176 of Graduate Texts in Mathematics. Springer-Verlag, New York, 1997. An introduction to curvature.

[20] Jean Leray. Sur le mouvement d'un liquide visqueux emplissant l'espace. Acta Math., 63(1):193-248, 1934.

[21] Anna L. Mazzucato. Besov-Morrey spaces: function space theory and applications to nonlinear PDE. Trans. Amer. Math. Soc., 355(4):1297-1364, 2003.

[22] Marius Mitrea and Michael Taylor. Navier-Stokes equations on Lipschitz domains in Riemannian manifolds. Math. Ann., 321(4):955-987, 2001.

[23] Seiki Nishikawa. Variational problems in geometry, volume 205 of Translations of Mathematical Monographs. American Mathematical Society, Providence, RI, 2002. Translated from the 1998 Japanese original by Kinetsu Abe, Iwanami Series in Modern Mathematics.

[24] Volker Priebe. Solvability of the Navier-Stokes equations on manifolds with boundary. Manuscripta Math., 83(2):145-159, 1994.

[25] Giovanni Prodi. Un teorema di unicità per le equazioni di Navier-Stokes. Ann. Mat. Pura Appl. (4), 48:173-182, 1959.

[26] John Roe. Elliptic operators, topology and asymptotic methods, volume 395 of Pitman Research Notes in Mathematics Series. Longman, Harlow, second edition, 1998.

[27] R. Schoen and S.-T. Yau. Lectures on differential geometry. Conference Proceedings and Lecture Notes in Geometry and Topology, I. International Press, Cambridge, MA, 1994. Lecture notes prepared by Wei Yue Ding, Kung Ching Chang [Gong Qing Zhang], Jia Qing Zhong and Yi Chao Xu, Translated from the Chinese by Ding and S. Y. Cheng, Preface translated from the Chinese by Kaising Tso.

[28] James Serrin. The initial value problem for the Navier-Stokes equations. In Nonlinear Problems (Proc. Sympos., Madison, Wis, pages 69-98. Univ. of Wisconsin Press, Madison, Wis., 1963.

[29] Dennis Sullivan. The Dirichlet problem at infinity for a negatively curved manifold. J. Differential Geom., 18(4):723-732 (1984), 1983. 
[30] Michael E. Taylor. Partial differential equations. III, volume 117 of Applied Mathematical Sciences. Springer-Verlag, New York, 1997. Nonlinear equations, Corrected reprint of the 1996 original.

[31] Roger Temam and Shou Hong Wang. Inertial forms of Navier-Stokes equations on the sphere. J. Funct. Anal., 117(1):215-242, 1993.

[32] Tai-Peng Tsai. On Leray's self-similar solutions of the Navier-Stokes equations satisfying local energy estimates. Arch. Rational Mech. Anal., 143(1):29-51, 1998.

[33] A. F Vasseur. Higher derivatives estimate for the 3D Navier-Stokes equation. ArXiv e-prints, April 2009.

[34] Shing Tung Yau. Harmonic functions on complete Riemannian manifolds. Comm. Pure Appl. Math., 28:201-228, 1975.

[35] Qi S. Zhang. The ill-posed Navier-Stokes equation on connected sums of $\mathbf{R}^{3}$. Complex Var. Elliptic Equ., 51(8-11):1059-1063, 2006.

Department of Applied Mathematics, National Chiao Tung University, 1001 Ta Hsueh Road, Hsinchu, Taiwan 30010, ROC

E-mail address: cchan@math.nctu.edu.tw

Department of Mathematical Sciences, Binghamton University (SUny), BinghamTON, NY 13902-6000, USA

E-mail address: czubak@math.binghamton.edu 